\title{
Aufbau und quasistationärer Zustand einer leistungsstarken toroidalen Pindh-Entladung in Wasserstoff *
}

\author{
Von U. Grossmann-Doerth \\ Aus dem Max-Planck-Institut für Physik und Astrophysik, München \\ (Z. Naturforschg. 16 a, 1290-1312 [1961] ; eingegangen am 5. Juli 1961) \\ Herrn Professor Werner Heisenberg zum 60. Geburtstag gewidmet
}

\begin{abstract}
Die Vorgänge in einer leistungsstarken elektrodenlosen Ringentladung in Wasserstoff wurden untersucht. Es zeigte sich, daß die Phänomene im Anfang der Entladung, insbesondere die Überführung des neutralen Gases in ein praktisch vollständig ionisiertes Plasma, mit einfachen Theorien zu verstehen sind. Nach Erreichen vollständiger Ionisation tritt ein quasistationärer Zustand ein, in dem das Plasma einen starken Energieverlust erleidet, der die Temperatur praktisch konstant auf etwa $5 \mathrm{eV}$ hält. Als Verlustprozesse kommen nur Instabilitäten in Frage.
\end{abstract}

Die toroidale Gasentladungsapparatur T-1 wurde Mitte 1957 konzipiert mit dem Ziel zu untersuchen:

1. Bis zu welchem Grad ist es möglich, in einer leistungsstarken elektrodenlosen Kondensatorentladung Wasserstoff zu ionisieren und aufzuheizen.

2. In welchem Maße läßt sich das magnetohydrodynamische („Pinch“-) Modell anwenden, in dem der Gasdruck und die Eigenkraft des Stromes $\mathfrak{i} \times \mathfrak{B}$ sich das Gleichgewicht halten, ohne daß Teilchen oder Energie verlorengehen.

In einer kurzen Abhandlung ${ }^{1}$ war abgeschätzt worden, daß vollständige Ionisation und Temperaturen von der Größenordnung $10^{6}{ }^{\circ} \mathrm{K}$ zu erwarten seien, falls keine besonderen Energieverluste auftreten.

Der Aufbau der Apparatur, zusammen mit allen Meßeinrichtungen, dauerte von Ende 1957 bis Ende 1959. Die während dieser Zeit durchgeführten, vorläufigen Messungen zeigten schon, daß die Plasmatemperatur etwa eine Größenordnung kleiner ist als erwartet. $\mathrm{Zu}$ ähnlichen Ergebnissen kamen die an anderen Stellen durchgeführten Untersuchungen des „z-Pinches“ 2, deren Resultate großenteils 1958 auf

* Dissertation der naturwissenschaftlichen Fakultät der Ludwig-Maximilians-Universität, München.

1 U. Grossmann-Doerth, nicht veröffentlicht.

2 Lineare oder toroidale Gasentladungen, in denen die Wechselwirkungskraft $\mathfrak{j} \times \mathfrak{B}$ des Entladungsstromes mit seinem eigenen Magnetfeld die wesentliche, das Plasma zusammenhaltende Kraft darstellt.

3 I. P. Conner, D. C. Hagerman, J. L. Honsaker, H. J. KarR, J. P. Mize, J. E. Osher, J. A. Phillips u. E. J. Stovall JR., Proc. Geneva Conf. 32, 297 [1958].

4 S. A. Colgate, J. P. Ferguson u. H. P. Furth, Proc. Geneva Conf. 32, 129 [1958].

5 S. A. Colgate, J. P. Ferguson, H. P. Furth u. R. E. Wright, Proc. Geneva Conf. 32, 140 [1958]. der Genfer und 1959 auf der Konferenz in Uppsala vorgelegt worden sind ${ }^{3-12}$.

Die vorliegende Arbeit enthält die Auswertung der endgültigen Meßserien. Die experimentellen Ergebnisse werden mit theoretischen Vorstellungen verglichen, um im Hinblick auf die beiden obigen Fragen eine möglichst geschlossene Beschreibung des Entladevorgangs zu erreichen.

\section{Die Entladungsanordnung}

\section{Der Torus}

Das eigentliche Entladungsgefäß ist ein horizontalliegender Torus aus Pyrexglas (Wandstärke 2,5 mm, kleiner Durchmesser $4 \mathrm{~cm}$, großer Durchmesser $100 \mathrm{~cm}$ ) mit einem vertikalen Stutzen $(\phi 1,5 \mathrm{~cm})$ für den Gaseinla $\beta$ und einem horizontalen Pumpstutzen $(\phi 3 \mathrm{~cm})$ auf der gegenüberliegenden Seite. Der Torus besteht aus zwei Hälften mit geschliffenen Stirnflächen, die mit Araldit zusammengekittet wurden, nachdem die Lage des Torus fixiert war.

\section{Der Kupfermantel}

Der Glastorus ist umgeben von einem Mantel aus Cu-Blech (2 mm stark) mit einem Schlitz (einige zehntel $\mathrm{mm}$ breit) in der Äquatorebene und einem Schlitz

6 T. E. Allibone, D. R. Сhick, G. P. Thomson u. A. A. W $\mathbb{W}_{\mathrm{Are}}$, Proc. Geneva Conf. 32, 169 [1958].

7 E. P. Butt, R. Carruthers, J. T. D. Mitchell, R. S. Pease, P. C. Thonemann, M. A. Bird, J. Blears u. E. R. Hartill, Proc. Geneva Conf. 32, 42 [1958].

8 A. A. Ware, Inst. El. Eng., Conv. Therm. Proc., Paper 2898 (April 1959)

9 R. M. Payne u. S. Kaufman, Inst. El. Eng., Conv. Therm. Proc., Paper 2900 (April 1959).

10 R. J. Bickerton, Inst. El. Eng., Conv. Therm. Proc., Paper 2883 (April 1959).

11 J. L. Tuck, Proc. Uppsala Conf., 920 (1959).

12 A. A. Ware, Proc. Uppsala Conf., 931 (1959). 
in der Vertikalebene (etwa $1 \mathrm{~cm}$ breit). An letzterem sind die Kondensatorzuführungen mit dem Cu-Mantel verbunden (vgl. Abb. 1). Der Cu-Mantel dient als Pri-

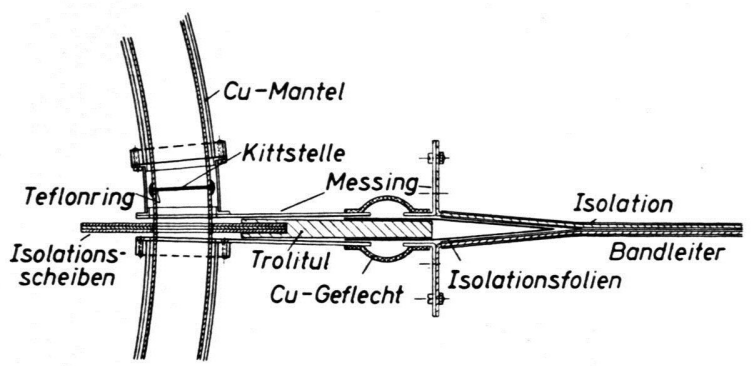

Abb. 1. Verbindung des Bandleiters mit dem Cu-Mantel.

märleiter eines 1 : 1-Transformators, dessen Sekundärschleife das Plasma im Glasgefäß ist. Außerdem soll er die Entladung stabilisieren (im Sinne der z. B. von R. J. TAYLER entwickelten Theorie). Diese elektrisch leitende Wand ist jedoch an mehreren Stellen unterbrochen, an der schon erwähnten Stelle, wo die Zuleitungen mit dem Mantel verbunden sind, an den beiden Pumpstutzen und an den 3 Beobachtungsschlitzen. Letztere sind relativ schmal (1-2 mm breit). Der Innendurchmesser des Cu-Torus beträgt knapp $6 \mathrm{~cm}$.

\section{Die elektrischen Bauteile des Entladekreises}

Als Leitung von der Kondensatorbatterie zum CuMantel diente anfangs ein Koaxialleiter, später eine in Araldit vergossene Bandleitung (Standardausführung des MPI). Als Schalter wurde ein Ignitron (Typ AJ 5551, AEG) benutzt.

Die Kondensatorbatterie besteht aus 20 parallelgeschalteten Bosch-Kondensatoren, deren Kapazität zusammen $154 \mu \mathrm{F}$ betrug. Die Batterie wurde maximal auf $10 \mathrm{kV}$ aufgeladen (Energieinhalt $8000 \mathrm{Ws}$ ).

Um Primär- und Sekundärkreis der Entladung möglichst gut elektromagnetisch zu koppeln, wurden 118 Ringbandkerne ( V a c u m s chmelze $\mathrm{Hanau}$, Querschnitt $1 \times 5 \mathrm{~cm}^{2}$, Banddicke $0,1 \mathrm{~mm}$, Material Trafoperm N 2, Grenzfrequenz $25 \mathrm{kHz}$ ) längs des Torusumfangs verteilt aufgestellt. Als sich zeigte, daß die Kerne weit vor Beendigung der Entladung in die Sättigung kamen, wurde ein großer Jochkern aus normalem Trafoblech (Dynamoblech 4\% Si, Querschnitt $30 \times 15 \mathrm{~cm}^{2}$, Blechdicke $0,35 \mathrm{~mm}$, Grenzfrequenz etwa $5 \mathrm{kHz}$ ) zusätzlich eingebaut. Der gesamte Spannungshub $U \cdot t=2 B_{\max } F$ beträgt (bei Vormagnetisierung) etwa $0,25 \mathrm{Vs}$, wie man z. B. der Abb. 2 entnehmen kann (Oszillogramm einer Entladung, in der das Gas erst zündete, als die Eisenkerne praktisch schon gesättigt waren). Um die Eisenkerne voll auszunutzen, werden sie vor jeder Entladung mit einer Hilfsentladung vormagnetisiert. Hierzu wird, durch die vorausgegangene Entladung gesteuert, eine kleine Batterie $(1000 \mu \mathrm{F})$ auf $900 \mathrm{~V}$ aufgeladen und über eine die Kerne umfassende Schleife entladen. Ein automatisch betätigter, mechanischer Schalter trennt diese Schleife während der Entladung von dem Vormagnetisierungskreis.

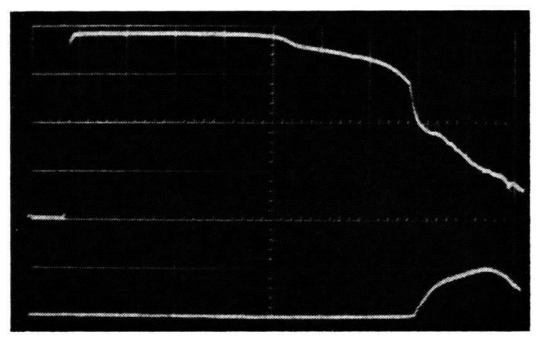

Abb. 2. Oszillogramm zur Bestimmung der Eigenschaften des Eisenkerns. Oberer Strahl: $U, 1 \mathrm{kV} / \mathrm{cm}$, unterer Strahl: $J_{\mathbf{2}}$, $5 \mathrm{kA} / \mathrm{cm}, 10 \mu \mathrm{s} / \mathrm{cm}$. Hier, wie in allen folgenden Oszillogrammen, wird der Abstand zwischen zwei Geraden des Koordinatennetzes "1 cm" genannt, entsprechend dem wirklichen

Abstand dieser Linien auf dem Oszillographenschirm.

\section{Das Stabilisierungsfeld}

Ein seelenparalleles Magnetfeld ( $B_{z}$-Feld“) wurde in vielen Fällen der Entladung überlagert. Es wird erzeugt durch Entladung einer Hilfsbatterie $(2000 \mu \mathrm{F}$, maximal $1000 \mathrm{~V}$ ) über ein längs des ganzen Torus auf den Cu-Mantel aufgewickeltes Solenoid. Den zeitlichen Verlauf des Stromes durch das Solenoid zeigt das Oszillogramm Abb. 3. Die Hauptentladung wurde kurz vor dem Strommaximum gezündet. Während ihrer Dauer (knapp $100 \mu \mathrm{s}$ ) ist das $B_{z}$-Feld praktisch konstant. $B_{z}$ kann variiert werden zwischen 0 und $800 \Gamma$. Der äquatoriale Schlitz im Cu-Mantel erlaubt ein ungehindertes Eindringen des $B_{z}$-Feldes in den Torus. Auf der anderen Seite kann man mit Konstanz des magnetischen Flusses innerhalb der Spule während der Zeit von einigen Mikrosekunden rechnen, weil die Zeitkonstante des Solenoids, $L / R$, sehr viel länger ist (Induktivität $L$ des Solenoids $0,2 \mathrm{mH}$, Widerstand $R=2 \Omega$, also $L / R=100 \mu \mathrm{s})$.

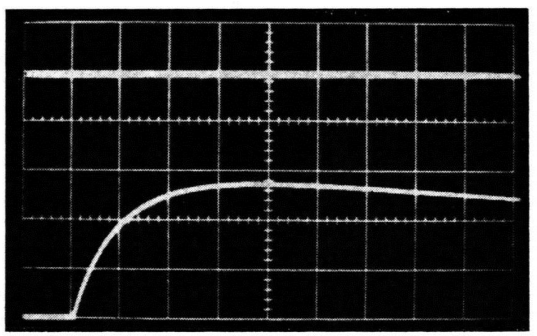

Abb. 3. Oszillogramm des $B_{z}$-Feld-Stromes $78 \mathrm{~A} / \mathrm{cm}$ ( $B_{z}=400 \Gamma \mathrm{im}$ Strommaximum). $100 \mu \mathrm{s} / \mathrm{cm}$.

Inhomogenitäten im $B_{z}$-Feld sind bedingt durch Unregelmäßigkeiten der Wicklung, die an den Stutzen, an den Beobachtungsfenstern und an der Stelle der Stromzuführung nicht zu vermeiden sind. Die Abweichung vom Sollwert des Magnetfeldes ist schätzungsweise nicht größer als $20 \%$. 


\section{Die Vorionisierung}

$\mathrm{Zu}$ Beginn der meisten Entladungen wurde das Gas im Torus schwach vorionisiert durch einen $27 \mathrm{MHz} \mathrm{HF}$ Impuls (Dauer etwa $100 \mu \mathrm{s}$ ), den ein $2 \mathrm{~kW}$-Sender erzeugt. Der Sender ist mit Hilfe einer dreiwindigen Spule um den Pumpstutzen an den Torus angekoppelt. Der HF-Impuls bringt den Wasserstoff im Pumpstutzen zum Leuchten; die Leuchterscheinung erstreckt sich, schwächer werdend, teilweise in den Torus.

Diese Vorionisierung vermindert den Zündverzug der Hauptenladung, besonders bei kleinen Spannungen und ganz niedrigen Drucken. Ein Einfluß auf den Verlauf der Entladung wurde nicht beobachtet.

In einer der Meßserien sollte untersucht werden, wie das dynamische Verhalten des kontrahierenden Plasmas sich ändert, wenn das Gas merklich vorionisiert wird (Kap. V). Zu diesem Zweck wurde der Hauptentladung eine Vorentladung vorausgeschickt. Der positive Pol einer der 20 Kondensatoren der großen Batterie wurde von der gemeinsamen Stromschiene getrennt; dieser Kondensator $(7,7 \mu \mathrm{F})$ wurde gesondert aufgeladen und dann mit Hilfe eines eigenen Ignitrons vor Beginn der Hauptentladung über den Cu-Mantel entladen. Das Oszillogramm einer Vorentladung allein zeigt Abb. 4, das einer Vorentladung mit Hauptentladung Abb. 16. Eine Induktivität von etwa $1 \mu \mathrm{H}$ begrenzt den Strom, der beim Zünden der Hauptentladung den kleinen Kondensator über das noch leitende Ignitron wieder auflädt.

\section{Blockschema und Ersatzschaltbild der Entladungsanordnung}

In dem Blockschema Abb. 5 sind die in diesem Kapitel beschriebenen Teile des Entladungskreises eingezeichnet; der Übersicht halber wurde nur einer der 118 kleinen Eisenkerne und nur ein Teil der $B_{z}$-Feldwicklung gezeichnet. Ein Teil des Torusumfangs ist vergrößert dargestellt.

Abb. 6 zeigt das Ersatzschaltbild der Entladung. Solange der Eisenkern nicht gesättigt ist, ist $L_{\mathrm{q}}$ hinreichend groß, um seinen Einfluß auf den Entladungsvorgang vernachlässigen zu können - Primär- und Sekundärstrom sind dann praktisch identisch. $L_{\mathrm{z}}$ stellt hauptsächlich die Induktivität des Ignitrons dar. Die primäre Streuinduktivität ist in der vorliegenden Geometrie verschwindend klein und wurde daher nicht eingezeichnet. $U$, die „Ringspannung“, ist die Spannung, die mit einer Schleife längs des Torus' gemessen wird.

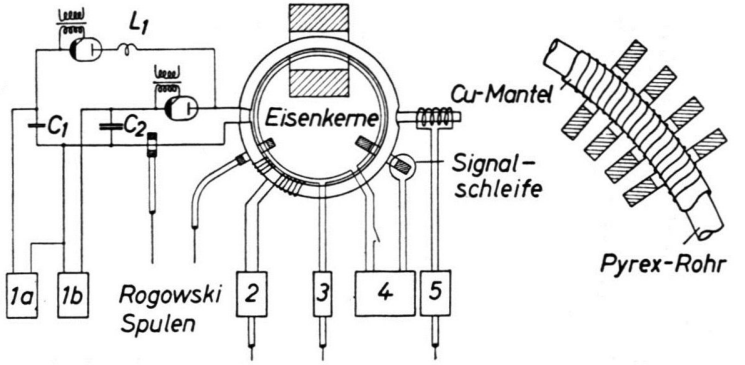

Abb. 5. Blockschema der Entladungsanordnung. 1 Ladegeräte, $2 B_{z}$-Feldgenerator, 3 Spannungsteiler, 4 Gerät zur autom. Vormagnetisierung, 5 Gepulster Sender zur Vorionisierung, $C_{1}$ Kondensator für die Vorentladung, $C_{2}$ Hauptentladungsbatterie, $L_{1}$ Schutzinduktivität.

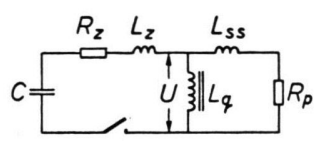

Abb. 6. Ersatzschaltbild des Entladekreises. $R_{\mathrm{z}}, L_{\mathrm{z}}$ Онмscher Widerstand bzw. Induktivität der Zuleitungen zum Torus, $L_{\mathrm{ss}}$ sekundäre Streuinduktivität, $R_{\mathrm{p}}$ OHmscher Widerstand des Plasmas, $L_{\mathrm{q}}$ Querinduktivität.

\section{Gaseinlaß und Vakuumsystem}

Alle hier beschriebenen Entladungen wurden in Wasserstoff durchgeführt, der mittels Elektrolyse erzeugt und durch ein geheiztes Pd-Röhrchen in den Torus eingelassen wurde. Der Gasfluß durch das Pd-Rohr betrug maximal $6 \cdot 10^{-2}$ Torr $l / s$. Vor jeder Entladung wurde der Torus ausgepumpt, bzw. mit durchströmendem Wasserstoff gespült. Mit Hilfe zweier magnetisch gesteuerter Ventile am Eingangs- und Ausgangsstutzen ließ sich der gewünschte Druck einstellen und der Torus abschließen. Im allgemeinen wurde der Torus vor Beginn einer Entladungsserie mit einer Vorpumpe ( L e y bold FD 2) auf einige $10^{-3}$ Torr evakuiert. Der Druckanstieg beträgt dann etwa $3 \cdot 10^{-5}$ Torr $l / \mathrm{s}$. Nach einer Belüftung wurde der Torus mit einer Öl-Diffusionspumpe ( L e y bold DO 30) mindestens $12 \mathrm{~h}$ ausgepumpt. Der Restgasdruck betrug dann $1-2 \cdot 10^{-5}$ Torr bei etwa $2 \cdot 10^{-5}$ Torr $l / s$ Druckanstieg. Die Arbeitsdrucke wurden mit einem LKB-Pirani-Gerät gemessen, das unter den vorliegenden Bedingungen eine Genauigkeit von etwa $10 \%$ haben soll (vgl. Laborbericht Nr. 6 der Vakuum-Abteilung, MPI, 1960). Der

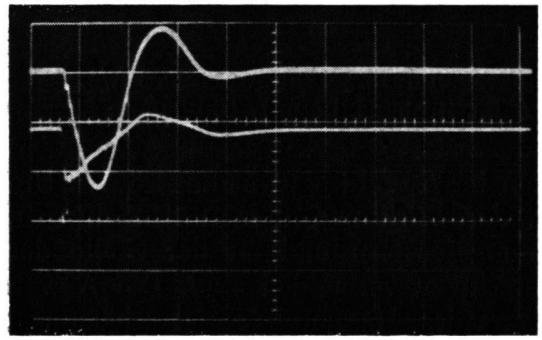

$\boldsymbol{a}$

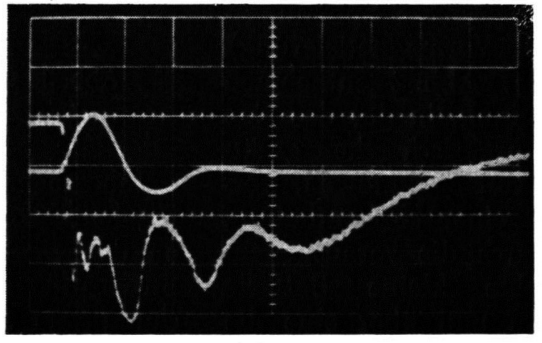

b
Abb. 4. Oszillogramm einer Vorentladung $\mathrm{i}\left(U_{00}=10 \mathrm{kV}, 0,1\right.$ Torr, $0 \Gamma$ ). a) Oberer Strahl (nach unten) : $J_{2}, 5 \mathrm{kA} / \mathrm{cm} ; 10 \mu \mathrm{s} / \mathrm{cm}$; unterer Strahl (nach oben): $U$, $5 \mathrm{kV} / \mathrm{cm}$; b) oberer Strahl (nach unten): $H_{\beta}$-Intensität; $10 \mu \mathrm{s} / \mathrm{cm}$; unterer Strahl (nach oben): $J_{1}$, $10 \mathrm{kA} / \mathrm{cm}$. 
Vergleich mit einem Le y b o ld - Alphatron zeigte $1 \%$ Differenz bei 0,1 Torr und 0,2 Torr, $10 \%$ bei 0,4 Torr, $30 \%$ bei 0,05 Torr und $60 \%$ bei 0,02 Torr.

\section{Steuerung der Entladung}

Der zeitliche Ablauf der Entladung wird von einem zentralen Impulsgeber gesteuert, aus dessen Kanälen zu den gewünschten Zeiten Spannungsimpulse kommen und die einzelnen Vorgänge der Entladung auslösen. Hierbei werden die beiden langen Verzögerungszeiten, zur Auslösung des $B_{z}$-Feldstromes $(t=350 \mu \mathrm{s})$ und zur Steuerung der Entladung von der Drehspiegelkamera aus ( $t$ bis zu $1000 \mu \mathrm{s}$ ) von zwei Transistor-Univibratoren hergestellt, während die folgenden, relativ kurzen Verzögerungszeiten (bis zu $100 \mu \mathrm{s}$ ) von einem Sägezahngenerator mit nachgeschalteten Diskriminatoren (MPI 5003/6) produziert werden. Das Steuergerät stand zusammen mit den Oszillographen in einer $\mathrm{Ab}$ schirmkabine (s. u.). Um zu verhindern, daß elektrische Störungen über die Steuerleitung in die Kabine kommen, mußten die Steuerkreise von der Außenwelt vollkommen entkoppelt werden. Hierzu wurden in der Kabine kleine Hilfssender aufgestellt, die, von den Steuerimpulsen angestoßen, monochromatische (10 MHz) Wellenpakete über abgeschirmte Leitungen nach außen schickten. Diese HF-Impulse werden in Bandfiltern gleichgerichtet, in denen Primär- und Sekundärkreis sehr sorgfältig kapazitiv entkoppelt sind. Die Ausgangsimpulse laufen in die störungsverseuchten Geräte, wie Hochspannungsimpulsgeber für die Zündung der Ignitrons usw. und lösen den gewünschten Vorgang aus ${ }^{13}$. Die zeitlichen Schwankungen der einzelnen Impulse waren nicht größer als etwa $2 \mu$ s (bei einer Gesamtverzögerungsdauer von $1000 \mu \mathrm{s}$, also $2 \%$ Unsicherheit). Der Zündverzug der Ignitrons betrug etwa $10 \mu$ s und schwankte um etwa $1 \mu$ s.

Die relativ langsamen Vorgänge des Entladungszyklus (Öffnen der Oszillographenkameras, Vormagnetisieren der Eisenkerne, Aufladen der Kondensatorbatterie) werden von relaisgesteuerten Geräten ausgelöst.

\section{Die Meßapparaturen}

\section{Störungsrauschen und Abschirmung}

Aus der Entladung kommt eine starke HF-Strahlung (Frequenzband von etwa $1 \mathrm{MHz}$ bis mindestens zur oberen Grenzfrequenz der Oszillographen, etwa $30 \mathrm{MHz}$ ), die eine extrem starke Abschirmung aller Meßleitungen notwendig machte. Aus diesem Grund wurden die Oszillographen zusammen mit dem zentralen Steuerimpulsgeber und einigen Versorgungsgeräten für die Photomultiplier in einer Abschirmkabine aufgestellt. Die Leitungen nach außen sind dreifach abgeschirmt ( $\mathrm{Siemens} \& \mathrm{Halske} 2 \mathrm{YC}(\mathrm{mS}) \mathrm{CY}$ $0,5 / 3,0)$ oder in $\mathrm{Cu}$-Rohren verlegt, wobei die äußeren Abschirmungen bzw. die Cu-Rohre mit der Kabine ver-

13 Die Anordnung geht auf einen Vorschlag von K.-H. SснмIтTER zurück. lötet sind. Jede Leitung aus der Kabine ist sozusagen eine fingerartige Erweiterung des abgeschirmten Raumes. Kopplungen, auch kapazitive, mit außenstehenden Geräten wurden sorgfältig vermieden.

\section{Die elektrischen Ströme}

Die Ströme wurden mit Rogovski-Spulen und nachfolgenden $R C$-Integratoren gemessen. Die untere Grenzfrequenz der Anordnung ist durch die Zeitkonstante $\tau$ der Integratoren gegeben und betrug 40 bzw. $20 \mathrm{~Hz}$ $(\tau=4 \mathrm{~ms}$ bzw. $8 \mathrm{~ms}$ ). Die Eigenfrequenz der RogovskiSpulen (einige $\mathrm{MHz}$ ) stellt die obere Grenzfrequenz für die Messung dar. Die Spulen sind mit einem niederohmigen Dämpfungswiderstand belastet. Eine Spule umfaßte eine Kondensatorzuführung und maß dementsprechend den Primärstrom $I_{1}$. Eine zweite Spule um den Cu-Mantel maß $I_{2}-I_{1}$ ( $I_{2}$ Strom im Plasma). Auf die beiden Eingänge eines Differenzenverstärkers (Tektronix G-Einschub) gegeben, lieferten die beiden Signale zusammen ein zu $I_{2}$ proportionales Signal.

\section{Ringspannung}

Die Ringspannung $U$ wurde mit einem OHмschen Spannungsteiler untersetzt, dessen kleiner Widerstand (75 $\Omega$ ) gleichzeitig als Kabelabschlußwiderstand diente. Die trotz Kompensation noch vorhandene Streukapazität der Widerstandskette ist die Ursache für eine obere Grenzfrequenz, die bei $3 \mathrm{MHz}$ lag.

\section{Kondensatorspannung}

Die Spannung $U_{\mathrm{c}}$ am Kondensator wurde mit einem kapazitiven Teiler gemessen. Um den Störpegel möglichst klein zu halten, wurden die beiden Ausgänge des Teilers mit den Eingängen eines Differenzverstärkers verbunden (das Prinzip, das Innere der Kabine galvanisch nicht mit einem Außenkreis zu verbinden, mußte hier durchbrochen werden). Die noch vorhandene HF auf dieser Leitung wurde mit einem Tiefpaß (obere Grenzfrequenz $4 \mathrm{MHz}$ ) weitgehend unterdrückt.

\section{Photomultiplier}

Die RöNTGEN-Strahlung sowie die Intensität vom gesamten sichtbaren Licht oder der $\mathrm{H}_{\beta}$-Strahlung wurde mit Photomultipliern gemessen (im Falle der RöNTGENStrahlung mit vorgesetztem Szintillationskristall, vgl. ${ }^{14}$ ). Die Photomultiplier waren, zusammen mit der nachfolgenden Elektronik, in Abschirmzylindern (Messing) untergebracht, die mit den Cu-Rohren verschraubt waren, in denen die notwendigen Versorgungs- und Signalleitungen liefen. Ein Blockschema der Meß-, Steuer- und Versorgungsleitungen zeigt Abb. 7.

\section{Oszillographen}

Die elektrischen Signale aus den Meßapparaturen wurden mit 3 Zweistrahloszillographen (Tektronix, Typ 551) aufgezeichnet. Die Oszillographenschirme

14 J. Junker, Diplomarbeit, Max-Planck-Institut für Physik und Astrophysik, München 1960. 


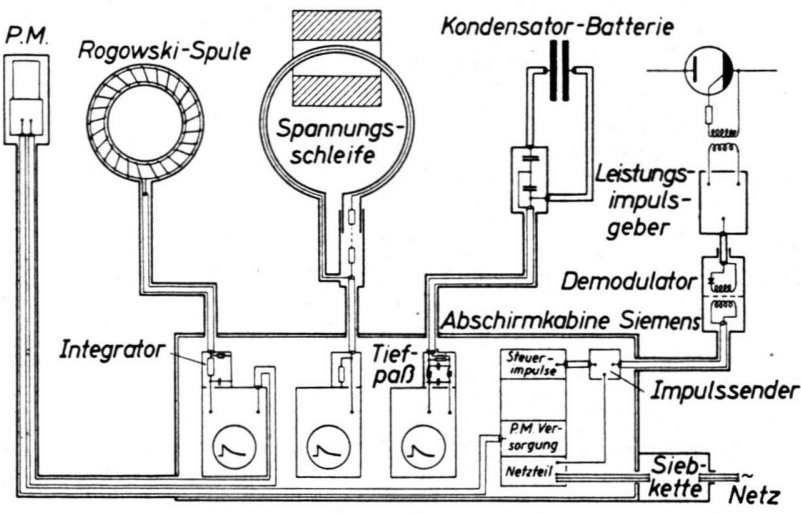

Abb. 7. Schema der Meß- und Steueranordnung.

wurden mit automatischen Kameras (Robot-Recorder) photographiert.

\section{Drehspiegelkamera}

Zeitlich aufgelöste Bilder der Entladung wurden mit einer Drehspiegelkamera ${ }^{15}$ aufgenommen, die das Bild eines vertikalen Schlitzes ( $1 \mathrm{~mm}$ breit) im Cu-Mantel über den Film laufen läßt. Unmittelbar vor dem Objektiv (Öffnung $1: 1,9 ; f=40 \mathrm{~mm}$ ) einer Robot-Kamera rotierte der Spiegel (Durchmesser $\sqrt{2}$-mal größer als der Objektivdurchmesser) mit maximal $800 \mathrm{~Hz}$ (Antrieb durch einen Kollektormotor). Das Öffnungsverhältnis der Kamera wird also durch den Spiegel nicht verändert. Im Abstand $115 \mathrm{~cm}$ vom Torus aufgestellt, hatte die Kamera eine zeitliche Auflösung (Zeit, die das Schlitzbild braucht, um sich um seine eigene Breite $\mathrm{zu}$ verschieben) von $0,17 \mu \mathrm{s}$ bei $400 \mathrm{~Hz}$ und $0,085 \mu$ s bei $800 \mathrm{~Hz}$. Die Breite des Schlitzbildes $(35 \mu)$ war nicht viel größer als der Korndurchmesser der photographischen Schicht - die Kamera war also optimal ausgenutzt. Die Synchronisierung der Entladung mit der Drehspiegelkamera erfolgte durch den Lichtstrahl eines Lämpchens, das über einen Hilfsspiegel auf der Drehachse zu einer bestimmten Zeit auf eine Photozelle fällt. Der dabei entstehende Impuls wird in das zentrale Steuergerät geleitet, wo er den Zeitpunkt Null auslöst. Eine bestimmte Zeit $t$ später wirft der Hauptspiegel das
Bild des Torusschlitzes auf den Filmrand - die Kamera „öffnet“. Gleichzeitig wird die Entladung gezündet. Die Zeit $t$ läßt sich durch Verdrehen des Hilfsspiegels auf der Achse einstellen.

\section{Parameterwerte}

Praktisch alle Entladungen wurden durchgeführt bei einer Kombination der folgenden Werte der drei Parameter :

Anfangsfülldruck im Torus $P_{0}=0,02 ; 0,05 ; 0,1$; 0,$2 ; 0,4$ Torr;

Ladespannung der Hauptbatterie $U_{0}=4,6,8,10 \mathrm{kV}$; Stabilisierungsfeld $B_{z}=0,400,800 \Gamma$.

\section{Der Entladungsvorgang}

Den zeitlichen Verlauf einer typischen Entladung geben die Oszillogramme der Abb. 8 wieder. Für einen quantitativen Vergleich mit theoretischen Vorstellungen ist es notwendig, die den Entladevorgang bestimmenden Größen zu ermitteln, nämlich induktiven und Oнмschen Widerstand des äußeren Kreises sowie Streuinduktivität und Oнmscher Widerstand des Plasmas (s. Ersatzschaltbild Abb. 6).

\section{Der äußere Kreis}

Der OHmsche Widerstand des äußeren Kreises, $R_{\mathrm{z}}$, ergab sich aus Dämpfungsmessungen zu $15 \mathrm{~m} \Omega$. Die Induktivität der Zuleitungen, $L_{\mathrm{z}}$, ist wesentlich durch das Ignitron bestimmt; sie wurde ermittelt aus dem Stromanstieg zu Beginn der Entladung $[\mathrm{d} I / \mathrm{d} t \sim$ $\left.\left(L_{z}+L_{\mathrm{ss}}\right)\right]$ und dem gleichzeitig gemessenen Wert der Ringspannung $\left[U \sim L_{\mathrm{ss}} /\left(L_{\mathrm{z}}+L_{\mathrm{ss}}\right)\right]$. Es ergab sich $L_{\mathrm{z}}=0,7 \mu \mathrm{H}$.

\section{Streuinduktivität des Plasmas}

Aus der Bestimmung von $L_{\mathrm{z}}$ folgte auch der Wert von $L_{\mathrm{ss}}$, der sekundären Streuinduktivität der Ent-

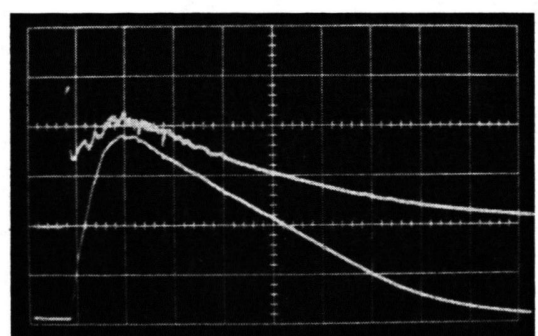

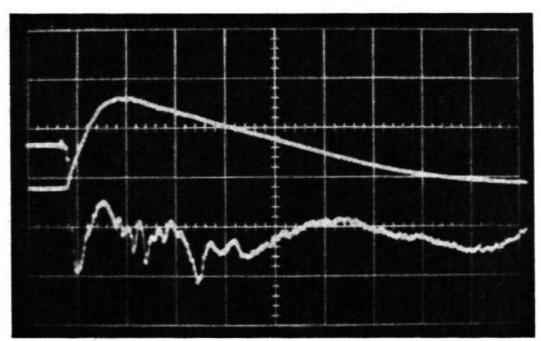

b

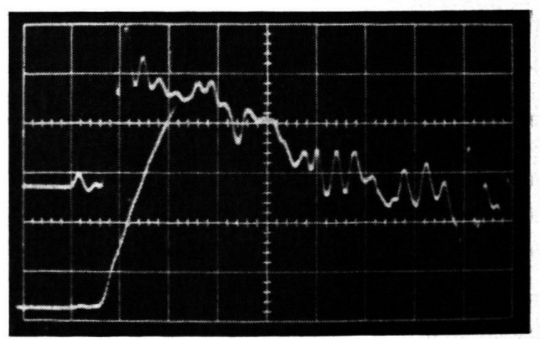

C

Abb. 8. Oszillogramme einer typischen Entladung $\left(U_{0}=6 \mathrm{kV}, P_{0}=0,05\right.$ Torr, $\left.B_{z}=800 \Gamma\right)$. a) Oberer Strahl: Ringspannung $U$, $2 \mathrm{kV} / \mathrm{cm}$; unterer Strahl: Plasmastrom $J_{2}, 5 \mathrm{kA} / \mathrm{cm} 10 \mu \mathrm{s} / \mathrm{cm}$. b) Oberer Strahl: Primärstrom $J_{1}, 10 \mathrm{kA} / \mathrm{cm} ;$ unterer Strahl: $H_{\beta}$-Intensität; $10 \mu \mathrm{s} / \mathrm{cm}$. c) Oberer Strahl: $\mathrm{d} J / \mathrm{d} t, 1,1 \cdot 10^{9} \mathrm{As}^{-1} / \mathrm{cm}$; unterer Strahl: $J_{1}, 2 \mathrm{kA} / \mathrm{cm} ; 2 \mu \mathrm{s} / \mathrm{cm}$.

15 Die Kamera wurde von E. REMY zur Verfügung gestellt. 
ladungsanordnung. Bei der im allgemeinen angewandten schwachen Vorionisierung beginnt der Plasmastrom nicht als Oberflächenstrom zu fließen, sondern ist über den ganzen Rohrquerschnitt verteilt. Daher ist die Induktivität des Plasmas gegen den Außenleiter nicht mehr frequenzunabhängig. Der nach der obigen Meßvorschrift ermittelte Induktivitätswert ist $0,7 \mu \mathrm{H}$. Berechnet man $L_{\mathrm{ss}}$ unter der Annahme von Oberflächenströmen in Abhängigkeit vom Plasmadurchmesser $r \quad\left(r_{0}=\right.$ Rohrdurchmesser $)$, so ergibt sich:

\begin{tabular}{|c|c|c|c|}
\hline$r / r_{0}$ & 1 & 0,75 & 0,5 \\
\hline$L_{\mathrm{ss}}$ in $\mu \mathrm{H}$ & 0,26 & 0,43 & 0,7 \\
\hline
\end{tabular}

Wie erwartet, ist der gemessene Wert von $L_{\mathrm{ss}}$ größer als der berechnete (das Plasma ist noch nicht kontrahiert z. Zt. der Messung von $\mathrm{d} I / \mathrm{d} t$ und $U$ ). Dagegen kann man aus der Tabelle abschätzen, daß das Meßresultat verträglich ist mit der Vorstellung von über den Rohrquerschnitt verteilten Volumenströmen. $L_{\mathrm{ss}}$ ist zeitlich nicht konstant (vgl. Kap. V). Man kann aus der Tabelle auch die ungefähren Grenzen sehen, innerhalb derer sich der Wert der Plasmainduktivität bewegen kann.

\section{Die elektrische Leitfähigkeit des Plasmas}

Der OHмsche Widerstand des Plasmas, $R_{\mathrm{p}}$, kann exakt nur z. Zt. des Strommaximums bestimmt werden, wenn die induktive Komponente der Ringspannung verschwindet (für $t=t_{\mathrm{m}}$ ist $R_{\mathrm{p}}=U / I$, wenn $t_{\mathrm{m}}$ der Zeitpunkt des Strommaximums ist). Hierbei muß noch vorausgesetzt werden, daß die Induktivität sich zeitlich nicht ändert. Dies ist gerechtfertigt, wenn man bedenkt, daß wegen der großen Skin-Tiefe merkliche Induktivitätsänderungen in Zeiten auftreten müßten, die nicht größer als etwa $1 \mu$ s sind. In der Tat sind der Spannungskurve Irregularitäten mit entsprechenden Frequenzen überlagert, die durch Induktivitätsschwankungen hervorgerufen sein könnten. Bei der Bestimmung von $U\left(t_{\mathrm{m}}\right)$ wurde aber über diese Zacken gemittelt. $R_{\mathrm{p}}\left(t_{\mathrm{m}}\right)$ liegt zwischen $0,15 \Omega$ und $0,45 \Omega$. Die Bedingung für eine überkritisch gedämpfte Kondensatorentladung, $4 L / R<$ $R C$, ist im allgemeinen erfüllt (wenn $R_{\mathrm{p}}>0,2 \Omega$ ) und dem entspricht der Verlauf des Entladungs-

${ }^{-16} \sigma_{\mathrm{p}}$ läßt sich (mit demselben Resultat) für $t>t_{\mathrm{m}}$ auch direkt aus Strom und Spannung bestimmen, weil der in- stromes: Ein Nulldurchgang des Stromes wird nur beobachtet, wenn der Eisenkern in die Sättigung kommt (dies tritt bei höherer Spannung ein). Die Leitfähigkeit des Plasmas wird also nach dem Strommaximum sicher nicht wesentlich größer.

Um zu prüfen, wie $R_{\mathrm{p}}$ sich im Verlauf der Entladung ungefähr ändert, wurde der gemessene Strom als Funktion der Zeit verglichen mit dem einer Kondensatorentladung mit zeitlich konstantem Widerstand und Induktivität. Der Vergleich führte zu dem Schluß, daß $\sigma_{\mathrm{p}}$, die elektrische Leitfähigkeit des Plasmas, sich zwischen etwa $10 \mu \mathrm{s}$ bis $60 \mu$ s nach Strombeginn nur um größenordnungsmäßig $10 \%$ ändert ${ }^{16}$. Die Meßwerte lassen sich von Entladung zu Entladung bis auf etwa 10\% reproduzieren.

In den Diagrammen der Abb. 9 ist $\sigma_{\mathrm{p}}$ als Funktion der drei Entladeparameter dargestellt. $\sigma_{\mathrm{p}}$ wurde aus $R_{\mathrm{p}}$ bestimmt unter der Annahme eines über den ganzen Rohrquerschnitt gleichmäßig verteilten Volumenstromes.

\section{Zeitlich aufgelöste Photographie der Entladung}

Das von einer Drehspiegelkamera aufgenommene Bild eines Vertikalschlitzes im Torusmantel zeigt, daß der ganze Rohrquerschnitt während etwa $1 \mu \mathrm{s}$ nach Beginn der Entladung schwach leuchtet. Die Leuchterscheinung verschwindet innerhalb von Bruchteilen einer $\mu$ s, wobei eine gerade noch zu erkennende konusartige Struktur auf eine Kontraktion des Plasmas hindeutet. Danach folgt eine etwa $1 \mu \mathrm{s}$ währende „Dunkelpause“ (vgl. Abb. 10 a) *. Ist ein $B_{z}$-Feld überlagert, laufen die Vorgänge langsamer ab und die ganze Struktur ist diffuser (vgl. Abb. $10 \mathrm{~b})$. Diese Erscheinungen während der ersten $\mu \mathrm{s}$ der Entladung sind gut reproduzierbar und treten bei fast allen Parameterwerten auf. Sie können erklärt werden durch Volumenströme, die das Gas schwach ionisieren und bei der Kontraktion z. Tl. mitnehmen (vgl. Kap. V). Die Dunkelpause ist vermutlich die Zeit, innerhalb derer der Strom in der Mitte des Torus kontrahiert ist. Der dabei komprimierte Wasserstoff ist vollständig ionisiert und leuchtet nicht mehr. Dann bricht die Konfiguration auf und die Elektronen ionisieren das restliche Gas.

Die auf die dunkle Periode folgenden Leuchterscheinungen sind in ihrer Struktur weniger regulär und von einer Entladung zur anderen verschie-

duktive Anteil der Spannung während des langsamen Stromabfalls klein ist.

* Abb. 10 a, b, c, d und 17 auf Tafel S. 1296 a. 


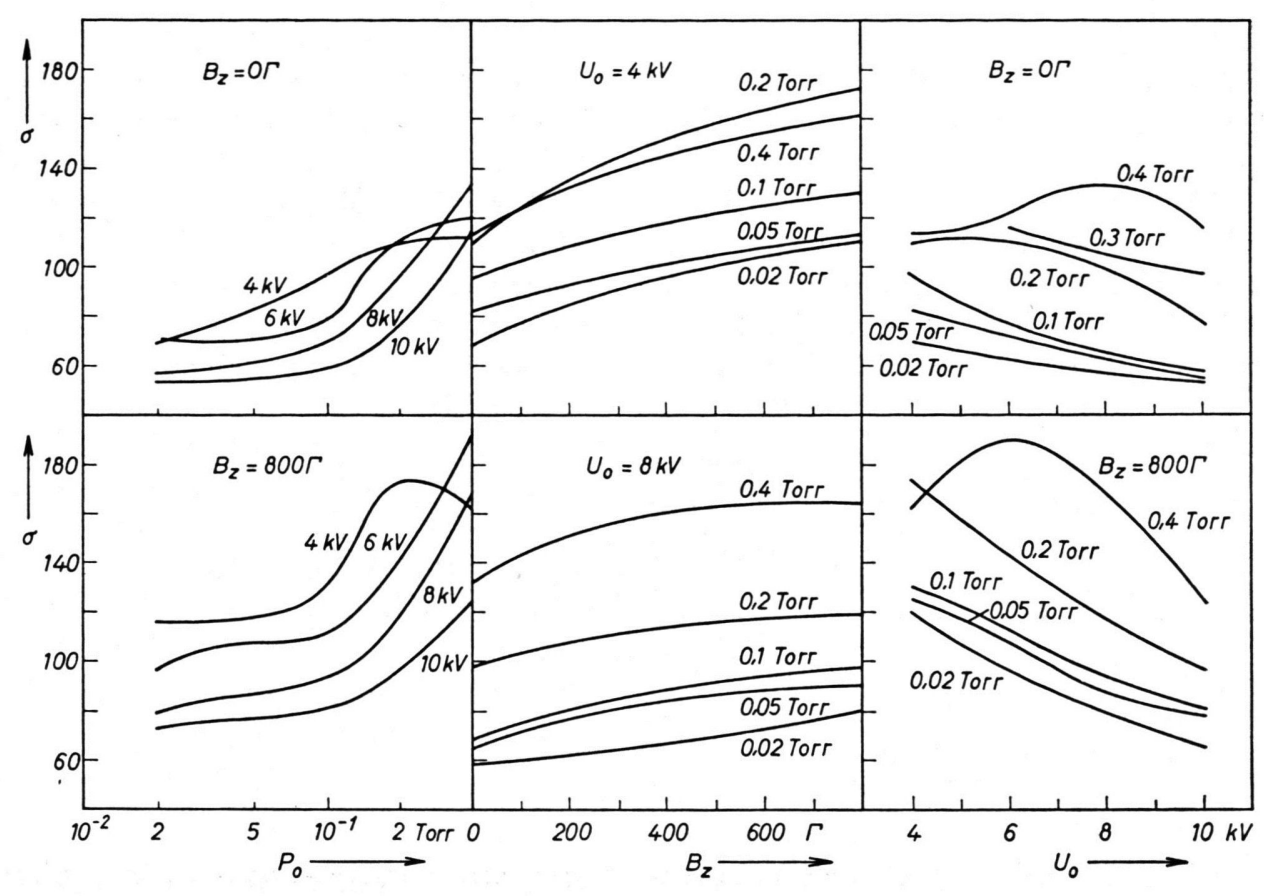

Abb. 9. Gemessene elektrische Leitfähigkeit in $\Omega^{-1} \mathrm{~cm}^{-1}$ des Plasmas zur Zeit des Strommaximums.

den; sie entsprechen den z. B. im SCEPTRE (Aldermaston) beobachteten (und dort barring genannten) Phänomenen (vgl. ${ }^{9}$ ). Um mehr über die leuchtenden Balken zu erfahren, wurde die Entladung durch einen horizontalen Schlitz photographiert (die Drehspiegelkamera war hierzu um $90^{\circ}$ gekippt). Eine weitere Aussage über den Charakter der Balken ließ sich aber aus diesen Bildern (vgl. Abb. $10 \mathrm{c}$ ) nicht gewinnen. Vermutlich geht die Bildstruktur auf Plasmaklumpen zurück, die intermittierend an die Wand geschleudert werden und dort sowohl kalt werden und selbst leuchten als auch Wandatome zum Leuchten anregen (vgl. ${ }^{8,12}$ ). Die Balken sind auch im Oszillogramm der Intensität der $H_{\beta}$-Linie zu erkennen (vgl. Abb. 8). Mit steigendem Druck und sinkender Spannung verlieren die Bilder an Struktur und sind schließlich weitgehend homogen (vgl. Abb. 10 d).

\section{Der Ionisierungsvorgang}

Schon während des Baues der Apparatur ist die Frage untersucht worden, ob man in einer Entladung der vorliegenden Art eine vollständige Ionisation des Gases erwarten könne ${ }^{1}$. Nachdem die ersten Meßergebnisse vorlagen, wurden die Berechnungen in einer veränderten, der Realität besser an- gepaßten Form wieder aufgenommen; ihr Hauptziel war, theoretische Aussagen zu gewinnen über den zeitlichen Verlauf der Elektronendichte. Die numerische Lösung der Gleichungen lieferte aber auch die Werte mehrerer anderer Größen als Funktion der Zeit, nämlich u. a. die elektrische Feldstärke im Plasma, den Plasmastrom und die Dichten der anderen auftretenden Teilchensorten. Ein Vergleich mit experimentellen Ergebnissen ist bei einigen Größen möglich und gibt Hinweise auf die Richtigkeit der zugrunde liegenden Annahmen.

\section{Das Gleichungssystem}

Das Gleichungssystem beschreibt den Verlauf einer Entladung unter den folgenden Voraussetzungen:

a) In dem Entladekreis ist nur der OHMsche Plasmawiderstand $R_{\mathrm{p}}$ zeitlich veränderlich.

Für den größten Teil der Entladung ist diese Annahme einer zeitlich konstanten Streuinduktivität gerechtfertigt, weil, wie gesagt, der OHMsche Widerstand des Plasmas erheblich größer ist als der induktive. Zu Beginn der Entladung dagegen, besonders wenn $B_{z}=0$ ist, treten Induktivitätsschwankungen auf (vgl. Kap. V), die merklichen Einfluß haben können. 
U. Grossmann-Doerth, Aufbau und quasistationärer Zustand einer leistungsstarken toroidalen Pinch-Entladung in Wasserstoff (S. 1290).
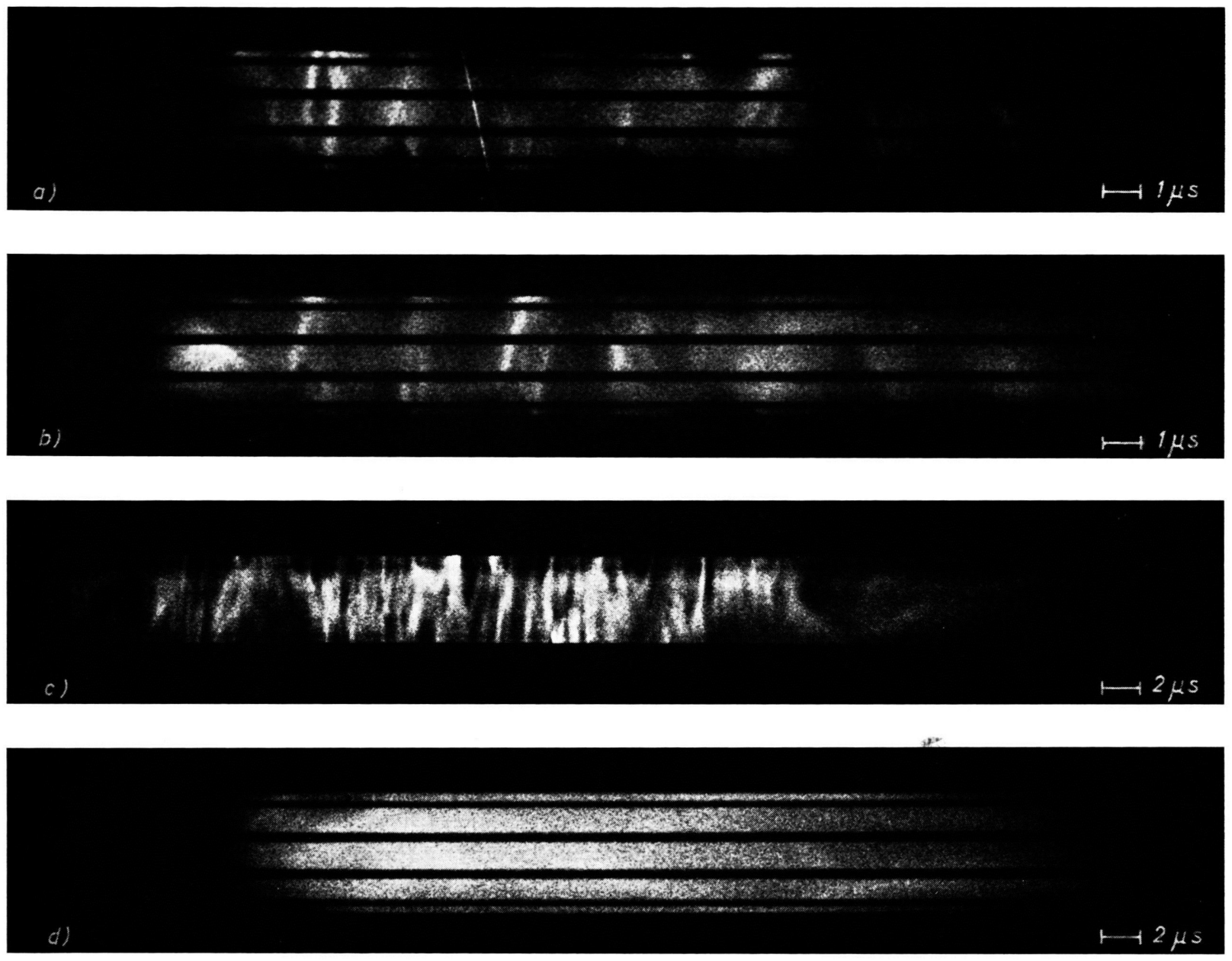

Abb. 10. Drehspiegelkamera-Aufnahmen der Entladung. Die Zeit läuft von links nach rechts. Das erste Aufleuchten kennzeichnet praktisch den Strombeginn. a) $8 \mathrm{kV}, 0,1$ Torr, $0 \Gamma$; vertikal (Beobachtungsschlitz senkrecht zur Torusebene). b) $8 \mathrm{kV}$ 0,1 Torr, $800 \Gamma$; vertikal. c) $8 \mathrm{kV}, 0,1$ Torr, $0 \Gamma$; horizontal (Beobachtungsschlitz in Torusebene). d) $4 \mathrm{kV}, 0,4 \mathrm{Torr}, 0 \Gamma$; vertikal.

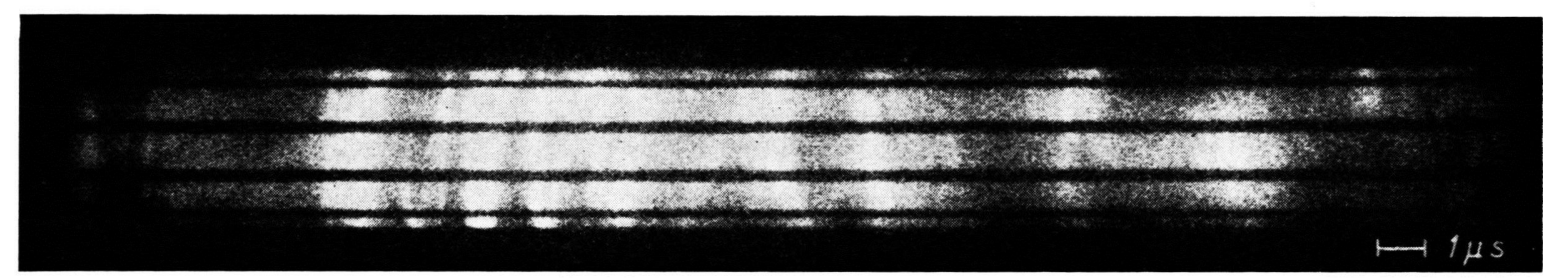

Abb. 17. Drehspiegelkamera-Aufnahme einer Entladung bei $8 \mathrm{kV}, 0,05$ Torr, $0 \Gamma$. Die Zeit läuft von links nach rechts. 

b) Der Strom hat räumlich konstante Dichte und erstreckt sich über den ganzen Rohrquerschnitt. Auch alle anderen Größen hängen nicht vom Ort ab. Insbesondere soll die elektrische Feldstärke im Plasma überall dieselbe sein.

Auch diese Annahmen sind zwar innerhalb der ersten Mikrosekunden nicht immer gut erfüllt, sonst aber im wesentlichen richtig.

c) Die Wirkungsquerschnitte für elastischen und unelastischen Elektronenstoß sowie die Diffusionskoeffizienten hängen nicht von der Zeit ab. Diese Vereinfachung erleichtert die Lösung des Gleichungssystems sehr. Auf der anderen Seite ändert sich die elektrische Feldstärke $F$ beträchtlich während des Ionisierungsvorganges; dabei bleibt auch $T_{\mathrm{e}}$, die Elektronentemperatur, nicht konstant und ebensowenig die Wirkungsquerschnitte für Elektronenstoß. Da für kleine Werte von $F / P_{0}\left(F / P_{0}<150 \mathrm{~V} / \mathrm{cm}\right.$ Torr $)$ z. B. der Ionisierungsquerschnitt stark von $F$ abhängt, kann die vorliegende Rechnung in solchen Fällen nur eine grobe Näherung sein. Schließlich sollte die Annahme ganz falsch sein nach Beendigung der Ionisation, wenn dem Plasma eine große elektrische Leistung (von der Ordnung $10^{8} \mathrm{~W}$ ) zugeführt wird und $T_{\mathrm{e}}$ daher stark steigen sollte. Die relativ gute Übereinstimmung zwischen gerechnetem und gemessenem Stromverlauf sollte einen interessanten Hinweis auf die Verlustprozesse geben.

d) Elektronen und Molekülionen diffundieren an die Wand, wo sie rekombinieren. Die dabei entstandenen $\mathrm{H}_{2}$-Moleküle erscheinen sofort, gleichmäßig verteilt, im Entladungsraum. Die Diffusionsgeschwindigkeit wird aus der Sснотткуschen Theorie abgeleitet. Somit wird die zeitliche Abnahme der Elektronendichte $n_{\mathrm{e}}$ infolge der (unter den vorliegenden Bedingungen ambipolaren) Diffusion beschrieben durch

$$
\frac{\mathrm{d} n_{\mathrm{e}}}{\mathrm{d} t}=-n_{\mathrm{e}} \frac{D_{\mathrm{a}}}{l^{2}}
$$

wobei $D_{\text {a }}$ der ambipolare Diffusionskoeffizient und $l$ eine charakteristische Länge von der Größenordnung der Gefäßdimension ist. Im vorliegenden Fall ist $l=r / 2,4 \approx 1(\mathrm{~cm}) \quad(r=$ Rohrradius $)$.

Für Ionisationsgrade wesentlich größer als $10 \%$ und für große Stromdichten gilt die Sснотткysche Theorie nicht mehr. Bei vollständiger Ionisation

17 L. Spitzer, jr., Physics of Fully Ionized Gases, Intersc. Publ., New York 1956.

18 Der Verlauf der Diffusion läßt sich im einzelnen den Glei- gelten die Plasmagleichungen des Zweiflüssigkeitsmodelles, die eine stationäre Lösung haben, in der keine Teilchen mehr aus dem Plasma herausdiffundieren (vgl. z. B. SpITzer ${ }^{17}$ ). Da der Übergang zwischen den beiden Grenzfällen stetig sein muß, folgt für die Diffusionsrate eine kontinuierliche $\mathrm{Ab}$ nahme von dem durch Gl. (1) gegebenen Wert nach Null ${ }^{18}$. Die Diffusionsverluste werden also überschätzt durch unsere Vereinfachung, daß Gl. (1) bis zur Erreichung des maximalen Ionisationsgrades gelten soll. Andere Teilchenverluste sollen nicht auftreten.

e) Alle Energieverluste sind enthalten in der Annahme, daß die Elektronentemperatur während der Entladung konstant bleibt trotz der dem Plasma zugeführten Energie.

Das Gleichungssystem besteht dann aus einer Gleichung für den Stromkreis (2), einer Gleichung für den Plasmawiderstand $R_{\mathrm{p}}(3)$ und 5 Bilanzgleichungen für die Dichten der verschiedenen Teilchenarten, Elektronendichte $\left(n_{\mathrm{e}}\right)$, Protonen $\left(n_{\mathrm{p}}\right), \mathrm{H}_{2}$ Moleküle $\left(n_{\mathrm{M}}\right)$, H-Atome $\left(n_{\mathrm{A}}\right)$ und Molekülionen $\left(n_{\mathrm{i}}\right):$

$\frac{1}{C} J+R_{\mathrm{p}} \frac{\mathrm{d} J}{\mathrm{~d} t}+J \frac{\mathrm{d} R_{\mathrm{p}}}{\mathrm{d} t}+L \frac{\mathrm{d}^{2} J}{\mathrm{~d} t^{2}}=0 ，$

$R_{\mathrm{p}}=\frac{K}{n_{\mathrm{e}}}\left(Q_{1} n_{\mathrm{M}}+Q_{2} n_{\mathrm{i}}+Q_{3} n_{\mathrm{p}}+Q_{4} n_{\mathrm{A}}\right)$

$\frac{\mathrm{l}}{n_{\mathrm{e}}} \frac{\mathrm{d} n_{\mathrm{M}}}{\mathrm{d} t}=-\left(\Sigma_{1}+\Sigma_{2}\right) n_{\mathrm{M}}+D_{\mathrm{a}}$,

$\frac{1}{n_{\mathrm{e}}} \frac{\mathrm{d} n_{\mathrm{A}}}{\mathrm{d} t}=2 \Sigma_{1} n_{\mathrm{M}}-\Sigma_{3} n_{\mathrm{A}}+\Sigma_{4} n_{\mathrm{i}}+\beta n_{\mathrm{p}}$,

$\frac{1}{n_{\mathrm{e}}} \frac{\mathrm{d} n \mathrm{e}}{\mathrm{d} t}=\Sigma_{2} n_{\mathrm{M}}+\Sigma_{3} n_{\mathrm{A}}+\Sigma_{5} n_{\mathrm{i}}-D_{\mathrm{a}}-\beta n_{\mathrm{p}}$,

$\frac{1}{n_{\mathrm{e}}} \frac{\mathrm{d} n_{\mathrm{i}}}{\mathrm{d} t}=\Sigma_{2} n_{\mathrm{M}}-\left(\Sigma_{4}+\Sigma_{5}\right) n_{\mathrm{i}}-D_{\mathrm{a}}$,

$\frac{1}{n_{\mathrm{e}}} \frac{\mathrm{d} n_{\mathrm{p}}}{\mathrm{d} t}=\Sigma_{3} n_{\mathrm{A}}+\left(\Sigma_{4}+2 \Sigma_{5}\right) n_{\mathrm{i}}-\beta n_{\mathrm{p}}$.

\section{Hierbei ist}

$C=$ Kapazität der Kondensatorbatterie,

$L=$ Induktivität des Entladekreises,

$K=$ geometrische Konstante,

$\Sigma=\left\langle v_{\text {th }} \sigma\right\rangle$ Reaktionsrate für inelastischen Elektronenstoß, gemittelt über die Geschwindigkeitsverteilung der Elektronen,

$v_{\text {th }}=$ thermische Geschwindigkeit der Elektronen,

$\sigma=$ unelastischer Streuquerschnitt,

chungen des Dreiflüssigkeitsmodells entnehmen, die von A. SCHLÜTER ${ }^{19}$ angegeben wurden.

19 A. Schlüter, Z. Naturforschg. 6 a, 73 [1951]. 
entsprechend

$Q=\left\langle v_{\text {th }} q\right\rangle$ mittlere Reaktionsrate für elastischen Elektronenstoß ( $q=$ elastischer Streuquerschnitt).

Im einzelnen bezieht sich

$\Sigma_{1}$ auf die Dissoziation des $\mathrm{H}_{2}$-Moleküls:

$\Sigma_{2}$ auf die Ionisation des $\mathrm{H}_{2}$-Moleküls: $\mathrm{e}+\mathrm{H}_{2} \rightarrow \mathrm{e}+2 \mathrm{H}$,

$\Sigma_{3}$ auf die Ionisation des H-Atoms: $\mathrm{e}+\mathrm{H}_{2} \rightarrow 2 \mathrm{e}+\mathrm{H}_{2}^{+}$,

$\Sigma_{4}$ auf die Dissoziation des Molekülions:

$\Sigma_{5}$ auf die Ionisation des Molekülions: $\mathrm{e}+\mathrm{H}_{2}^{+} \rightarrow \mathrm{e}+\mathrm{H}+\mathrm{H}^{+}$,

Entsprechend bezieht sich

$$
\mathrm{e}+\mathrm{H}_{2}^{+} \rightarrow 2 \mathrm{e}+2 \mathrm{H}^{+} \text {. }
$$

$Q_{1}$ auf den (elast.) Stoß der Elektronen mit

$\mathrm{H}_{2}$-Molekülen,

$Q_{2}$ auf den (elast.) Stoß der Elektronen mit $\mathrm{H}_{2}{ }^{+}$Ionen,

$Q_{3}$ auf den (elast.) Stoß der Elektronen mit Protonen,

$Q_{4}$ auf den (elast.) Stoß der Elektronen mit Atomen.

Weiterhin ist $\beta=$ Volumenrekombinationskoeffizient $\left(\mathrm{e}+\mathrm{H}^{+} \rightarrow \mathrm{H}\right)$ und $D_{\mathrm{a}}=$ Koeffizient der ambipolaren Diffusion.

\section{Die Konstanten}

Die Werte der atomaren Konstanten des Gleichungssystems sind vielfach nicht genau bekannt, woraus eine weitere Ungenauigkeit in unserem Ergebnis resultiert.

Zur Gewinnung der unelastischen Streuquerschnitte wurde zunächst ein während der Dauer des Ionisationsvorganges mittlerer Wert der elektrischen Feldstärke im Plasma $F$ aus Messungen und vorausgegangenen Rechnungen geschätzt. Aus $F / P_{0}$ ergab sich ein Wert für die Elektronentemperatur $T_{\mathrm{e}}$, bei Verwendung der in der Gasentladungsliteratur angegebenen Kurven $T_{\mathrm{e}}=f(F / P)$, vgl. z. B. v. ENGEL $^{20}$, BRown ${ }^{21}$. Mit diesem Wert von $T_{\mathrm{e}}$ wurden die $\Sigma$ bestimmt entsprechend den von Schmidt ${ }^{22}$ angegebenen Kurven. Um die diesem Verfahren anhaftenden Unsicherheiten abzuschätzen, wurde die besonders wichtige Ionisierungsrate der $\mathrm{H}_{2}$-Moleküle, $\Sigma_{2}$, verglichen mit den sich aus dem sog. Townsend-Koeffizienten $\alpha$ ergebenden Werten. $\alpha / P$ $=f(E / P)$ ist für Wasserstoff vielfach gemessen worden 20, 21, 23. Da für den Verlauf der Ionisation

20 A. v. Engel, Ionized Gases, Clarendon Press, Oxford 1955.

21 S. C. Brown, Basic Data of Plasma Physics, Chapman \& Hill, London 1959.

22 H. U. Sснміdт, in Vorbereitung.

23 L. B. Loeb, Basic Processes of Gaseous Electronics, Univ. Calif. Press, Berkeley 1955. durch Elektronenstoß gilt

$$
\frac{\mathrm{d} n_{\mathrm{e}}}{\mathrm{d} t}=\alpha n_{\mathrm{e}} v_{\mathrm{D}}=n_{\mathrm{e}} n_{\mathrm{M}} \Sigma_{2},
$$

läßt sich $\alpha$ mit $\Sigma_{2}$ vergleichen, wenn $v_{\mathrm{D}}$, die Driftgeschwindigkeit der Elektronen, bekannt ist. Für $v_{\mathrm{D}}$ wurde angenommen

$v_{\mathrm{D}}\left[\mathrm{cm} \mathrm{s}^{-1}\right]=4 \cdot 10^{5} \mathrm{~F} / P_{0} \quad\left(F\right.$ in $\mathrm{V} / \mathrm{cm}, P_{0}$ in Torr $)$ (vgl. $\left.{ }^{20}, 21,24,25\right)$. Damit ergibt sich das Diagramm Abb. 11. $\bar{\alpha}$ entstand durch Mittelung über die verschiedenen in der Literatur angegebenen Werte des Townsend-Koeffizienten. Die beiden Kurven weichen z. Tl. erheblich voneinander ab. Die gestrichelte Kurve wurde für die Rechnung benutzt.

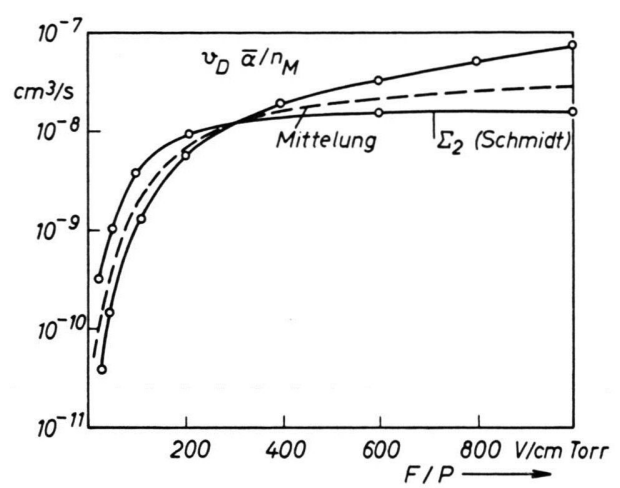

Abb. 11. Ionisierungsrate von $\mathrm{H}_{2}$-Molekülen infolge Elektronenstoß.

Für die elastischen Stoßraten der Elektronen mit Molekülen und Atomen wurde angenommen

$$
Q_{1}=Q_{4}=10^{-7} \mathrm{~cm}^{3} \mathrm{~s}^{-1}
$$

(die Wirkungsquerschnitte sind bei den kleineren Energien etwa umgekehrt proportional zur Geschwindigkeit); diese Zahl folgt aus dem oben genannten Wert für die Driftgeschwindigkeit $v_{\mathrm{D}}$. Die Größe des Elektron - Proton-Streuquerschnittes bestimmt während des größten Teils der Entladung die elektrische Leitfähigkeit des Plasmas. Daher wurde $Q_{3}$ dem gemessenen $R_{\mathrm{p}}$ angepaßt entsprechend der Beziehung

$\sigma_{\mathrm{p}}=\frac{e^{2} n_{\mathrm{e}}}{m \gamma}=\frac{e^{2} n_{\mathrm{e}}}{m n_{\mathrm{p}} Q_{3}}=\frac{2,8 \cdot 10^{-4}}{Q_{3}}$ in $\Omega^{-1} \mathrm{~cm}^{-1}$,

wobei $\gamma$ die Stoßfrequenz ist und $Q_{3}$ wieder in

24 W. L. Granovski, Der elektrische Strom im Gas, AkademieVerlag, Berlin 1955.

25 B. B. Rossi u. H. H. Staub, Ionisation Chambers and Coun ters, McGraw Hill, New York 1949. 
$\mathrm{cm}^{3} \mathrm{~s}^{-1}$ gemessen sein soll. Die Gleichung gilt für vollständige Ionisation. Ferner wurde $Q_{2}=Q_{3}$ angenommen.

Der ambipolare Diffusionskoeffizient $D_{\mathrm{a}}$ ist gegeben durch

$$
D_{\mathrm{a}}=\frac{D^{+}+D^{-}\left(K^{+} / K^{-}\right)}{1+K^{+} / K^{-}} .
$$

Hierbei sind $D$ die einfachen Diffusionskoeffizienten, $K$ die Beweglichkeiten; Index + bezieht sich auf Ionen, Index - auf Elektronen. Wenn der Entladung ein $B_{z}$-Feld überlagert war, wurden in dieser Formel für $D$ die Koeffizienten der Diffusion quer zu einem Magnetfeld

$$
D_{\perp}=\frac{D}{1+a(\omega / \gamma)^{2}}
$$

( $\omega=$ Gyrofrequenz, $\gamma=$ Stoßfrequenz, $\alpha=1$ für Elektronen, 0,25 für Ionen) und für $K$ die Beweglichkeiten quer zum Magnetfeld

$$
K_{\perp}=\frac{K}{1+a(\omega / \gamma)^{2}}
$$

eingesetzt.

Auch ohne $B_{z}$-Feld ist, zumindest nach einiger Zeit, das Magnetfeld des Entladungsstromes groß genug (im Strommaximum zwischen etwa 2000 und $4000 \Gamma$ ), um die Diffusion wesentlich zu vermindern. Auf der anderen Seite sollte der Einfluß der Diffusionsverluste dadurch abgeschätzt werden, daß in einigen Fällen die größten möglichen Diffusionsraten angenommen wurden.

Die Werte der Koeffizienten $D$ und $K$ wurden für jeden einzelnen Fall nach der Diffusionstheorie ausgerechnet.

Für $\beta$, den Koeffizienten der Volumenrekombination, wurde angenommen: $\beta=10^{-12} \mathrm{~cm}^{3} \mathrm{~s}^{-1}$ (vgl. z. B. Massey und Burhop ${ }^{26}$ ). Weil die Volumenrekombination klein ist gegenüber der Diffusion zur Wand bei den vorliegenden Bedingungen, wurde die Abhängigkeit des Koeffizienten von den Parameterwerten nicht berücksichtigt.

\section{Lösungen}

Das Gleichungssystem wurde für etwa 25, den Parameterbereich möglichst gut überdeckende Fälle numerisch gelöst. Als Beispiel zeigt Abb. 12 vier Lösungen.

In allen Fällen liefert die Rechnung praktisch vollständige Ionisation innerhalb von Zeiten, die

${ }^{26}$ H. Massey u. E. Burhop, Electronic and Ionic Impact Phenomena, Clarendon Press, Oxford 1952. meist zwischen 1 und $3 \mu$ s liegen, für die kleinsten $F / P_{0}$-Werte aber bis zu $10 \mu$ s reichen. Es zeigte sich, daß man die Zeit, innerhalb derer $\eta=0,5$ erreicht wird, $t_{50}$, aus einer einfachen Abschätzung in guter Näherung gewinnen kann. Gl. (6) läßt sich nämlich für $\eta<0,5$ vereinfachen zu

$$
\frac{\mathrm{d} n_{\mathrm{e}}}{\mathrm{d} t} \approx n_{\mathrm{e}} \Sigma_{2}\left(n_{\mathrm{M}}+n_{\mathrm{A}}\right) \approx n_{\mathrm{e}} n_{\mathrm{M} 0} \Sigma_{2} .
$$

Da mit $\eta_{0}=5 \cdot 10^{-5}$ gerechnet wurde, ist

$$
n_{\mathrm{e}}\left(t_{50}\right) / n_{\mathrm{e} 0}=10^{4},
$$

und es ergibt sich aus (13):

$$
\ln 10^{4}=n_{\mathrm{M} 0} \Sigma_{2} t_{50}, \quad t_{50}=\frac{9,2}{n_{\mathrm{M} 0} \Sigma_{2}}[\mathrm{~s}] .
$$

Der Index 0 bezieht sich auf $t=0$; der Ionisations$\operatorname{grad} \eta$ ist definiert durch $\eta=n_{\mathrm{e}} / 2 n_{\mathrm{M} 0}$.

Das so berechnete $t_{50}$ stimmt mit dem aus der Lösung des Gleichungssystems sich ergebenden Wert bis auf ungefähr $20 \%$ überein trotz der verschiedenen, bei dem Ionisierungsvorgang auftretenden Zwischenprodukte.

Die elektrische Feldstärke $F$ im Plasma ist gegeben durch $F=I R /(\pi D)=I R / 314$. Wie die Kurven der Abb. 12 zeigen, ändert sich $F$ während des Ionisationsprozesses nicht unwesentlich. Da auf der anderen Seite mit konstanten Wirkungsquerschnitten gerechnet wurde, liegt hier eine Inkonsequenz vor, auf die schon im vorletzten Abschnitt hingewiesen wurde. In Wirklichkeit werden sowohl Maximum wie Minimum von $F(t)$ weniger ausgeprägt sein, weil z. B. ein Wert von $F$, der größer ist als der der Rechnung zugrunde liegende, ein größeres $\Sigma_{2}$ und damit einen größeren Stromanstieg zur Folge hat; damit aber wird der induktive Spannungsabfall größer und $F$ wieder kleiner. Infolge dieses „Stabilisierungseffektes“ sind die tatsächlich auftretenden $\mathrm{Ab}$ weichungen der elektrischen Feldstärke von dem Mittelwert also kleiner als aus den Diagrammen zu folgern ist.

\section{Vergleich zwischen Theorie und Experiment}

\subsection{S tromver la u f}

Gerechneter und gemessener Stromverlauf können nur verglichen werden, solange die Sättigung des Eisenkerns noch keinen Einfluß hat, d. h. bei $U_{0}=$ $8 \mathrm{kV}$ nur bis etwa $50 \mu \mathrm{s}$, bei $6 \mathrm{kV}$ bis $70 \mu$ s und bei $4 \mathrm{kV}$ bis $90 \mu$ s nach Strombeginn.

Der gerechnete Stromanstieg während der ersten Mikrosekunden, solange die Ionisation noch nicht 

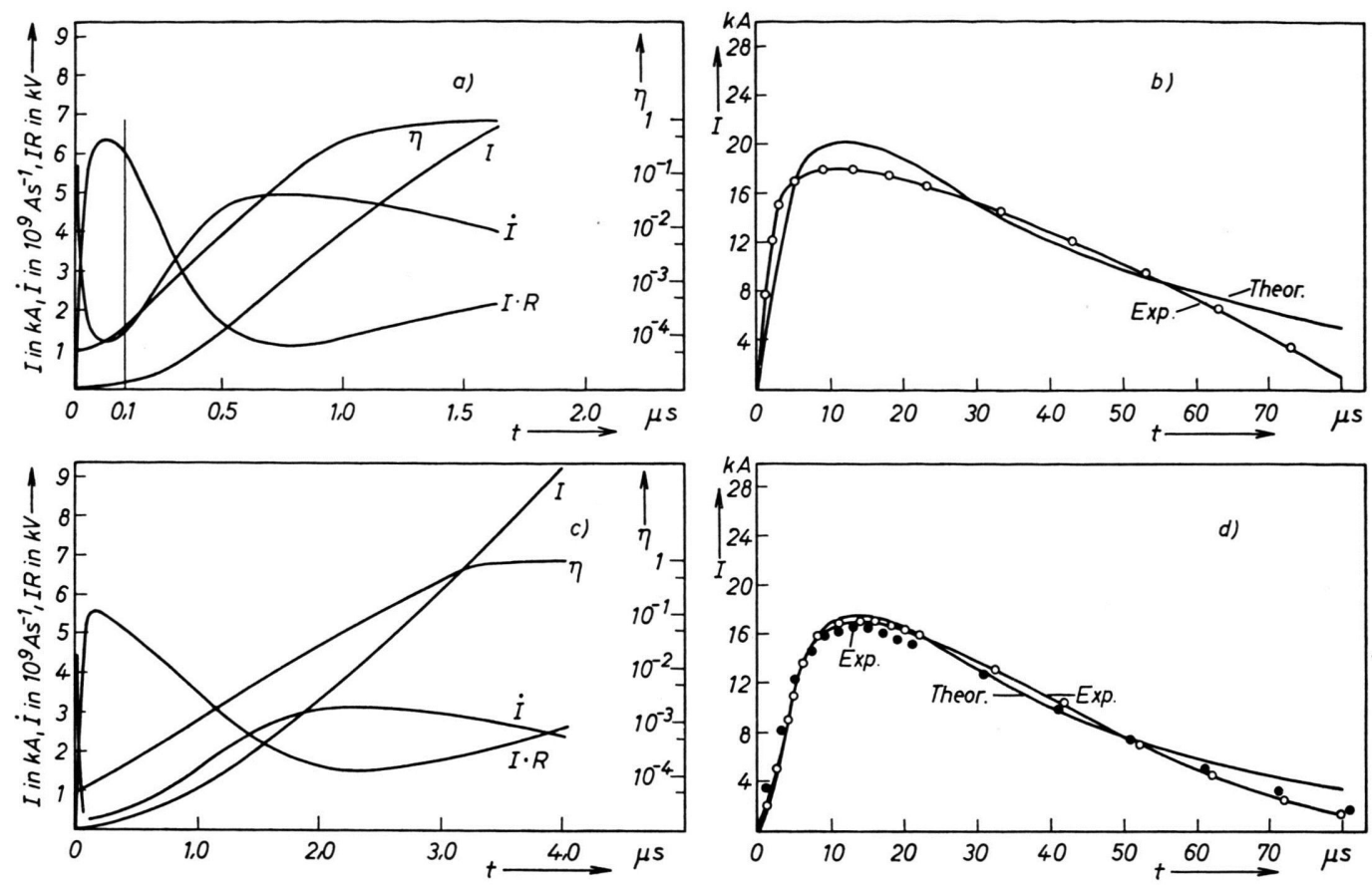

Abb. 12 a, b. Verlauf der Entladung bei $8 \mathrm{kV} ; 0,02$ Torr; $800 \Gamma$.

Abb. 12 c, d. Verlauf der Entladung bei $6 \mathrm{kV} ; 0,05$ Torr; $800 \Gamma$. $\circ$ und $\bullet$ gehören zu 2 verschiedenen Entladungen.
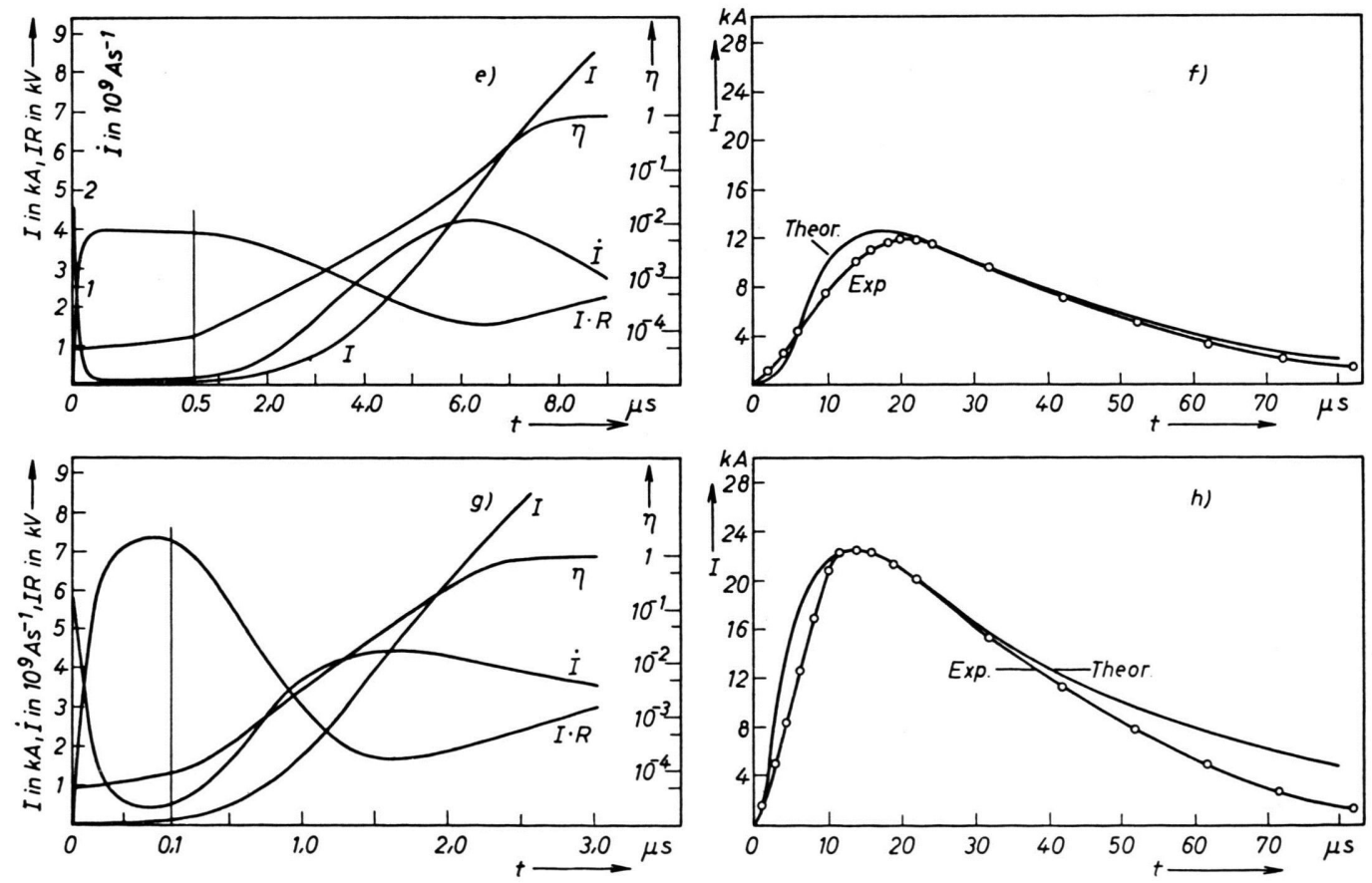

Abb. 12 e, f. Verlauf der Entladung bei $4 \mathrm{kV} ; 0,2$ Torr; $0 \Gamma$.

Abb. $12 \mathrm{~g}$, h. Verlauf der Entladung bei $8 \mathrm{kV} ; 0,2$ Torr; $0 \Gamma$. 
vollständig ist, stimmt mit den Meßwerten innerhalb der Fehlergrenzen überein, die durch die Unsicherheit in der Ionisationsrate (vgl. Kap. II) gegeben sind. Im weiteren Verlauf des Stromes treten Diskrepanzen bis zu 20\% auf, aber im allgemeinen sind die Abweichungen nicht größer als 5\%. Falls unser Modell richtig ist und sich nicht unberücksichtigte Effekte in ihrem Einfluß auf den Strom gegenseitig aufheben, kann gefolgert werden, daß der Wirkungsquerschnitt für elastische Streuung der Elektronen an Protonen und damit die Elektronentemperatur während einer relativ langen Zeit im wesentlichen konstant bleibt. Es bleibt die Frage, ob nicht andere Prozesse als die elastische Streuung der Elektronen an Protonen die elektrische Leitfähigkeit bestimmen. Eine Antwort werden erst die spektroskopischen Meßergebnisse bringen.

\subsection{Intensitätsverlauf der $\mathrm{H}_{\beta}$ - Linie}

Das Oszillogramm der Intensität der aus dem Torus kommenden $\mathrm{H}_{\beta}$-Linienstrahlung $(\lambda=4861 \AA)$ weist einige Mikrosekunden nach Strombeginn ein mehr oder weniger ausgeprägtes Maximum auf (s. Oszillogramm der Abb. 8b). Dieses wurde in $\mathrm{Zu}$ sammenhang gebracht mit dem Maximum, das die Linienstrahlung als Funktion der Elektronentemperatur $T_{\mathrm{e}}$ oder des Ionisationsgrades $\eta$ hat. Abb. 13 zeigt $\mathrm{H}_{\beta}$-Linenstrahlung und Ionisationsgrad in $\mathrm{Ab}$ hängigkeit von ${ }^{27} T_{\mathrm{e}}$. Wie man sieht, erreicht die Intensität der $\mathrm{H}_{\beta}$-Linie ungefähr bei $90 \%$ Ionisation ihr Maximum. Um einen Anhalt dafür zu gewinnen,

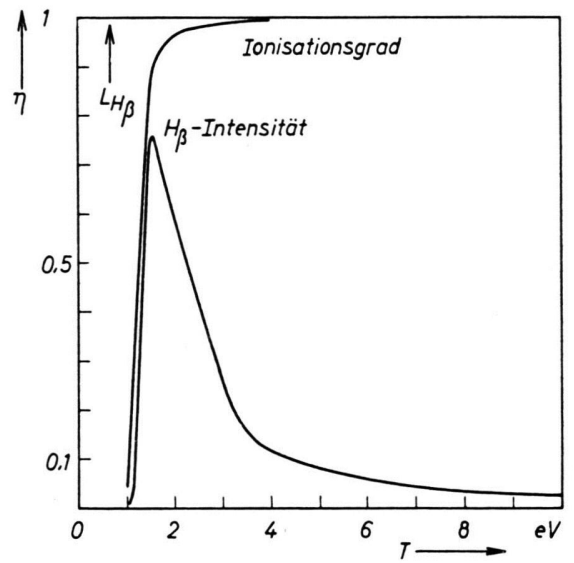

Abb. 13. Ionisationsgrad $\eta$ und Intensität der $\mathrm{H}_{\beta}$-Linie in relat. Einheiten (berechnet).

27 Die Kurven wurden gerechnet mit den der Koronaformel zugrunde liegenden Voraussetzungen, also dünnes Gas, ob der vermutete Zusammenhang besteht, werden in Tab. 1 die gemessenen Zeitpunkte maximaler $\mathrm{H}_{\beta}$ Strahlung verglichen mit den Zeiten, nach denen, entsprechend unseren Rechnungen, 90\% Ionisation erreicht sind. Die Übereinstimmung ist wieder innerhalb der Grenzen, die durch die Unsicherheit in der Ionisationsrate gegeben sind (besonders bei hohen Drucken und kleinen Spannungen ist die Ionisationsrate, und damit $t_{\mathbf{9 0}}$, nur bis auf einen Faktor 2-3 bekannt). Trotzdem könnte man die Tatsache, daß $t_{90}$ fast immer kleiner ist als $t_{\mathrm{M}}$, als einen Hinweis auf einsetzende Verlustprozesse werten.

\begin{tabular}{|c|c|c|c|l|}
\hline $\begin{array}{c}P_{0} \\
\text { Torr }\end{array}$ & $\begin{array}{c}U_{\mathbf{0}} \\
\mathrm{kV}\end{array}$ & $\begin{array}{c}B_{z} \\
\Gamma\end{array}$ & $\begin{array}{c}t_{\mathrm{M}} \\
\mu \mathrm{s}\end{array}$ & $\begin{array}{l}t_{90} \\
\mu \mathrm{s}\end{array}$ \\
\hline 0,02 & 4 & 800 & 2 & 2,5 \\
0,02 & 6 & 800 & 1 & 2,0 \\
0,02 & 8 & 800 & 0,5 & 1,4 \\
0,05 & 4 & 800 & 3 & 3 \\
0,05 & 6 & 800 & 3 & 1,5 \\
0,05 & 8 & 800 & 3 & 1,2 \\
0,1 & 4 & 0 & 7 & 4 \\
0,1 & 4 & 800 & 7 & 4 \\
0,1 & 8 & 0 & 3 & 2,5 \\
0,1 & 8 & 800 & 4 & 2,5 \\
0,2 & 4 & 0 & 16 & 7 \\
0,2 & 4 & 800 & 15 & 7 \\
0,2 & 8 & 800 & 6 & 4 \\
0,4 & 8 & 0 & 10 & 6 \\
0,4 & 8 & 800 & 7 & 6 \\
\hline
\end{tabular}

Tab. 1. Zeit des ersten beobachteten Maximums der $\mathbf{H}_{\beta}$ Linienstrahlung $t_{\mathrm{M}}$ und die theoretisch zu erwartende Zeit bis zur Erreichung von $90 \%$ Ionisation $t_{90}$.

Insgesamt vermuten wir aus dem in Tab. 1 durchgeführten Vergleich, daß vollständige Ionisation des Plasmas erreicht wird innerhalb von Zeiten, die vergleichbar sind mit den theoretisch zu erwartenden Werten.

Ein zweites, flaches Maximum der $\mathrm{H}_{\beta}$-Intensität wird beobachtet, wenn der Strom wieder sehr klein geworden ist, es ist ausgeprägter bei einem Nulldurchgang des Stromes. Dieses Maximum entspricht dann dem zweiten, diesmal von höheren Werten herkommenden Durchlaufen des Ionisationsbereiches um $90 \%$.

Zwischen diesen beiden Maxima ist die $\mathrm{H}_{\beta}$-Intensität nicht klein, sondern schwankt um einen Mittelwert, der vergleichbar ist mit dem Wert des ersten

keine Besetzung der Atomzustände nach Boltzmans, aber MAxwellsche Verteilung der Elektronengeschwindigkeiten. 
Maximums. Die Schwankungen sind irregulär, nicht reproduzierbar und treten in Abständen von größenordnungsmäßig $1 \mu$ s auf. Offenbar kühlt sich das Plasma in einer nicht ganz kontinuierlichen Weise ab - vermutlich durch Auftreffen auf die Wand. Dann wird es wieder ionisiert und aufgeheizt. Wir nehmen an, daß diese Teilchenverluste die Ursache dafür sind, daß die Elektronentemperatur trotz der großen Energiezufuhr nicht steigt (s. u.).

\subsection{Spektroskopische Untersuchungen}

A.m T-1 wurden von der spektroskopischen Gruppe des Instituts eine Reihe von Messungen durchgeführt, die in einem Laborbericht ${ }^{28}$ im einzelnen beschrieben sind, und deren Ergebnisse wir im folgenden wiedergeben.

Die Elektronendichte im Torus wurde, in Abhängigkeit von der Zeit, bei vier verschiedenen Parameterwerten bestimmt (aus der STARK-Verbreiterung der Balmer-Linien). Die Zeitauflösung der Meßapparatur betrug $5-10 \mu \mathrm{s}$, als Fehler wird $\pm 10 \%$ angegeben. Die Tab. 2 enthält die Meßresultate. Aus Intensitätsgründen ließ sich die Messung nicht auf kleinere Drucke ausdehnen.

\begin{tabular}{|c|c|c|c|c|c|c|c|c|c|c|}
\hline \multirow[b]{2}{*}{$\begin{array}{c}t \\
\mu \mathrm{s}\end{array}$} & \multicolumn{4}{|c|}{$\begin{array}{c}P_{0}=0,2 \text { Torr } \\
\left(n_{\mathrm{M}_{0}}=6 \cdot 10^{15} \mathrm{~cm}^{-3}\right)\end{array}$} & \multicolumn{6}{|c|}{$\begin{array}{c}P_{0}=0,4 \text { Torr } \\
\left(n_{\mathrm{M}_{0}}=12 \cdot 10^{15} \mathrm{~cm}^{-3}\right)\end{array}$} \\
\hline & 5 & 30 & 60 & 70 & 10 & 20 & 40 & 50 & 60 & 70 \\
\hline$B_{z}=0$ & - & 8,7 & 6,3 & 4,6 & - & 19 & 17 & 15 & 14 & 10 \\
\hline $\begin{array}{l}B_{z}= \\
800 \Gamma\end{array}$ & 9,1 & 7,8 & 7,2 & 7,5 & 20 & - & - & - & 13 & 8,1 \\
\hline
\end{tabular}

Tab. 2. Elektronendichte $n_{\mathrm{e}}$ in Einheiten von $10^{15} \mathrm{~cm}^{-3}$ als Funktion der Zeit $t$ nach Beginn der Entladung. $U_{0}=8 \mathrm{kV}$ in allen Fällen.

Die hier gemessenen Elektronendichten (Mittelwerte über $5 \mu \mathrm{s}$ ) erreichen offenbar noch vor dem Strommaximum ihren größten Wert, der einem Ionisationsgrad $\eta$ von $70-80 \%$ entspricht, wenn man $\eta=n_{\mathrm{e}} / 2 n_{\mathrm{M} 0}$ setzt $\quad\left(n_{\mathrm{M} 0}=\right.$ Anfangsdichte der Moleküle). $n_{\mathrm{e}}$ fällt dann langsam ab, ändert sich aber während der $50 \mu$ s, innerhalb derer wegen Sättigung des Eisenkerns ein Vergleich zwischen Theorie und Experiment nur möglich ist, nicht mehr als etwa $20 \%$.

Wir werten diese Meßergebnisse als Unterstützung der bisher entwickelten Vorstellungen, nach denen

28 K. Bergstedt u. H. WulfF, Unveröffentlichter Laborbericht des MPI, 1961. zu Beginn der Entladung vollständige Ionisation erreicht wird, dann aber eine starke Plasmadiffusion zur Wand auftritt, der durch eine Rückdiffusion der infolge Rekombination an der Wand entstandenen Moleküle eine Art von Gleichgewicht gehalten wird.

Die Elektronentemperatur $T_{\mathrm{e}}$ wurde aus dem Auftreten von O II-, O III- und O IV-Linien sowie aus dem Intensitätsverhältnis von O II- zu O III-Linien bestimmt. Bei allen vier Parameterwerten der Tab. 2 ergab sich $T_{\mathrm{e}}=5 \mathrm{eV} \pm 15 \%$. Der Wert gilt für Zeiten 10 bis $20 \mu$ s um das Strommaximum herum, eine genauere Zeitauflösung war wegen der geringen Intensität der Verunreinigungslinien nicht möglich.

Die Tab. 3 gibt zum Vergleich die Elektronentemperaturen, die gemäß der Formel von Spitzer ${ }^{17}$ aus der (im Strommaximum gemessenen) elektrischen Leitfähigkeit folgen. Der Einfluß des Magnetfeldes wurde berücksichtigt.

\begin{tabular}{|l|l|l|}
\hline$P_{0}$ Torr & 0,2 & 0.4 \\
\hline$B_{z}=0$ & 5,2 & 5,2 \\
$B_{z}=800 \Gamma$ & 5 & 6 \\
\hline
\end{tabular}

Tab. 3. Elektronentemperaturen in eV zur Zeit des Strommaximums. $U_{0}=8 \mathrm{kV}$ in allen Fällen.

Wir folgern aus der guten Übereinstimmung mit dem spektroskopisch bestimmten Wert von $T_{\mathrm{e}}$, daß die SpItzersche Leitfähigkeitsformel unter den vorliegenden Bedingungen Geltung hat. Die Verlustprozesse haben keinen großen Einfluß auf die elektrische Leitfähigkeit im Plasma. Die Elektronentemperatur bleibt wirklich, wie in unserem Modell angenommen, über relativ lange Zeiten im wesentlichen konstant.

Spektroskopische Momentaufnahmen des Torusquerschnittes ergaben bei einer Öffnungszeit von $10 \mu$ s keinen Hinweis auf eine radiale Abhängigkeit von Teilchendichten oder Elektronentemperatur.

Neben den Wasserstofflinien treten im Spektrum der Entladung einige schwache Linien auf, die von den folgenden ionisierten Atomen emittiert werden: O II $(35,2)$, O III $(54,9)$, O IV $(77,4)$, C II $(24,4)$, C III $(47,9)$, F II $(35,0)$, F III $(62,7)$, Si IV $(45,1)$; die Zahlen in Klammern geben die Ionisierungsspannung in eV an. Die Sauerstoff- und Siliciumionen kommen vermutlich aus der Glaswand, die Kohlenstoffionen aus dem Pumpenöl und die Fluorionen aus zwei Teflonringen (diese Ringe sitzen an den Kittstellen zwischen den beiden Torushälften, vgl. Abb. 1). Aus der Intensität dieser Linien wurde 
der Grad der Verunreinigung während der Entladung auf größenordnungsmäßig $1 \%$ geschätzt.

\section{Dynamik der Pinchbewegung}

\section{Experimentelle Ergebnisse und Theorie}

Dem zu Beginn der Entladung stetig ansteigenden Plasmastrom ist innerhalb der ersten Mikrosekunden eine gedämpfte Schwingung überlagert (vgl. Abb. 14), die in Frequenz und Amplitude von den Entladungsparametern abhängt und gut reproduzierbar ist. Gleichzeitig mit einem Schwingungsmaximum tritt eine scharfe Spitze in der Spannung $U$ auf; im Augenblick des ersten relativen Strommaximums erreicht $U$ praktisch den Wert $U_{0}$ (Ladespannung des Kondensators). Ein überlagertes $B_{z}$-Feld verringert die Amplitude der Schwingung stark. Die beobachtete Schwingung ist also sicher keine Eigenschwingung z. B. der Rogovski-Spulen. Es gibt zwei Erklärungsmöglichkeiten. Entweder der OHмsche Plasmawiderstand $R_{\mathrm{p}}$ oder die Streuinduktivität $L_{\mathrm{ss}}$ des Plasmas ändern sich periodisch. Da kein plausibler Grund bekannt ist für eine Änderung von $R_{\mathrm{p}}$ der erforderlichen Art, wurde diese Möglichkeit ausgeschlossen. Dagegen schien von vornherein das von der Stromkontraktion verursachte zeitliche Anwachsen von $L_{\mathrm{ss}}$ die Ursache für die Schwingung zu sein. Die sich in Richtung auf die Seele hin zusammenziehenden Stromfäden nehmen die ionisierte Materie mindestens teilweise mit (Pinch). Daher ist die Bewegung mit einer trägen Masse verknüpft, die bewirken kann, da $\beta \mathrm{d} L / \mathrm{d} t$ so groß wird, $\mathrm{da} \beta \mathrm{d} I / \mathrm{d} t<0$ werden muß, um die Gleichung

$$
U_{\mathrm{c}} \approx U_{0} \approx L_{\mathrm{z}} \frac{\mathrm{d} I}{\mathrm{~d} t}+L_{\mathrm{ss}} \frac{\mathrm{d} I}{\mathrm{~d} t}+I \frac{\mathrm{d} L_{\mathrm{ss}}}{\mathrm{d} t}+R I
$$

zu erfüllen (vgl. Ersatzschaltbild Abb. 6) .
Ein diese Zusammenhänge beschreibendes Gleichungssystem wurde von LEHNER aufgestellt und auf eine lineare $z$-Pinch-Entladung angewandt ${ }^{29}$. Es wird auf Leontovitch und Osovets ${ }^{30}$, auf die das Gleichungssystem zurückgeht, verwiesen. Ähnliche Untersuchungen wurden durchgeführt z. B. von PHILLIPS ${ }^{31}$, Bodin ${ }^{32}$ et al. und Latham et al. ${ }^{33}$. Hierbei sind die folgenden, vereinfachenden Annahmen gemacht worden:

a) Die Leitfähigkeit des Plasmas ist zeitlich konstant und unendlich, der Plasmastrom ist also stets ein reiner Oberflächenstrom.

b) Die von der stromführenden Zylinderfläche zusammengedrückten Ionen und Elektronen haben eine Geschwindigkeit $\dot{r} \sim r$; die Dichte des Gases innerhalb des Zylinders bleibt dann konstant.

c) Die Gastemperatur $T$ ist vom Radius $r$ unabhängig. $T$ variiert mit der Zeit entsprechend den - adiabatisch angenommen - Kompressionen und Expansionen.

LEHNER hat seine beiden Gleichungen numerisch gelöst und über eine, was die Zeitpunkte der Kontraktionen betrifft, sehr gute Übereinstimmung der Rechenergebnisse mit den experimentellen Beobachtungen beim schnellen linearen Pinch berichtet. Die Gleichungen wurden auf die vorliegende Torusentladung angewandt und numerisch gelöst unter der Annahme, daß man den Torus wie einen Zylinder der Länge $\pi D(D=$ Torusdurchmesser $)$ behandeln kann. Der Stromverlauf wurde ausgerechnet für 21 verschiedene Kombinationen der Parameter $U_{0}(4 \mathrm{kV}, 8 \mathrm{kV}), P_{0}(0,02,0,05,0,1$ Torr $), B_{z}$ $(0,400 \Gamma)$ und $\gamma$, dem „Mitnahmefaktor". der gleich dem Bruchteil, der sich an der Bewegung beteiligenden Masse ist. Die Abb. 15 zeigt als Beispiel einige gerechnete und gemessene Stromverläufe. Da die

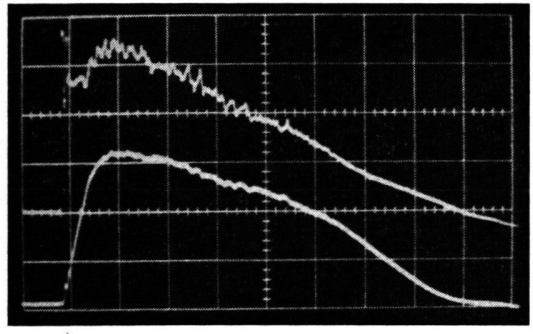

$\boldsymbol{a}$

29 E. Fünfer, G. Lehner u. H. Tuczek, Z. Naturforschg. 15 a, 566 [1960].

30 M. A. Leontovich u. S. M. Osovets, J. Nucl. Energy 4, 209 [1957].

31 N. J. Phillips, Proc. Uppsala Conf., 849 (1959).
Abb. 14. Pinch-Oszillationen. Entladung bei $8 \mathrm{kV}, 0,05$ Torr, $0 \Gamma$. a) Oberer Strahl: $U, 2 \mathrm{kV} /$ $\mathrm{cm}$; unterer Strahl: $J_{2}, 5 \mathrm{kA} / \mathrm{cm}$; $10 \mu \mathrm{s} / \mathrm{cm}$. b) Oberer Strahl: $\mathrm{d} I / \mathrm{d} t, 2,2 \cdot 10^{9} \mathrm{As}^{-1} / \mathrm{cm}$; unterer Strahl: $J_{1}, 1 \mathrm{kA} / \mathrm{cm} ; 1 \mu \mathrm{s} / \mathrm{cm}$.

$$
\text { b }
$$

32 H. A. B. Bodin, N. J. Peacock u. J. A. Reynolds, Proc. Uppsala Conf. 861 [1959].

33 R. Latham, F. L. Curzon u. J. A. Nation, Proc. Uppsala Conf. 884 [1959]. 


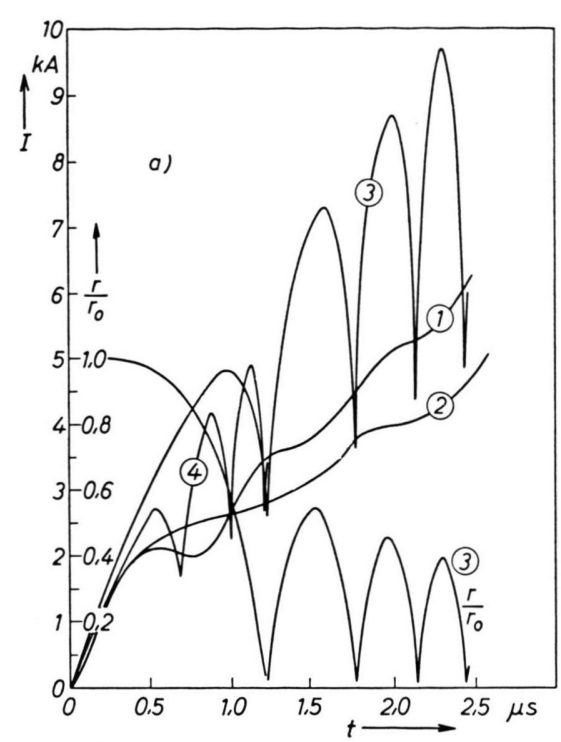

Abb. 15 a. Gemessener und berechneter Stromverlauf sowie berechneter Verlauf des relativen Pinchdurchmessers $r / r_{0}$ für $8 \mathrm{kV}$; 0,02 Torr; 0 T. 1 Experimentell, ohne Vorentladung; 2 experimentell, mit Vorentladung; 3 theoretisch, $\gamma=1$; 4 theoretisch, $\gamma=0,1$.

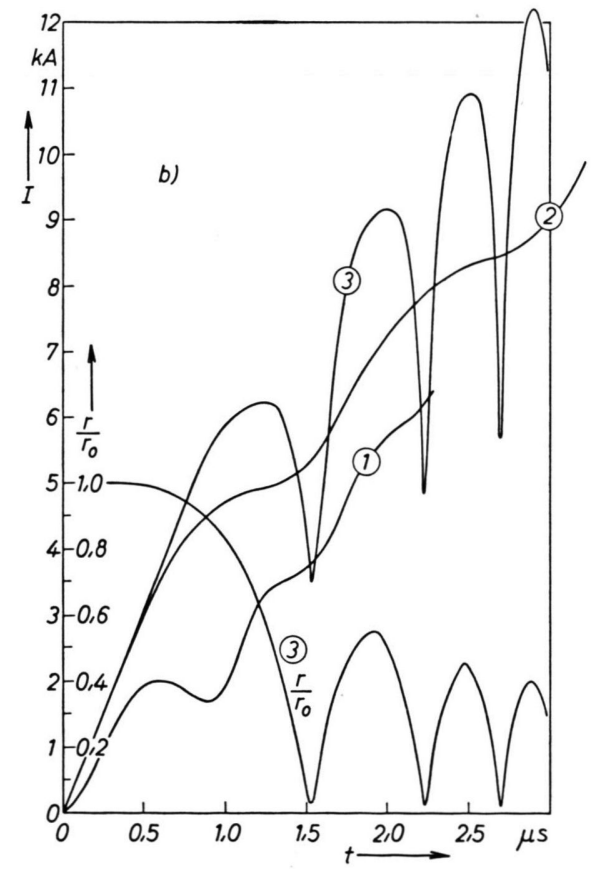

Abb. 15 b. Gemessener und berechneter Stromverlauf sowie berechneter Verlauf des relativen Pinchdurchmessers $r / r_{0}$ für $8 \mathrm{kV}$; 0,05 Torr; $0 \Gamma$. 1 Experimentell, ohne Vorentladung; 2 experimentell, mit Vorentladung; 3 theoretisch, $\gamma=1$.

Diskrepanzen zwischen Messung und Rechnung z. Tl. darauf zurückgeführt worden sind, $\mathrm{da} ß$ der Ionisationsgrad und damit die an der Kontraktionsbewegung möglicherweise sich beteiligende Masse bei der

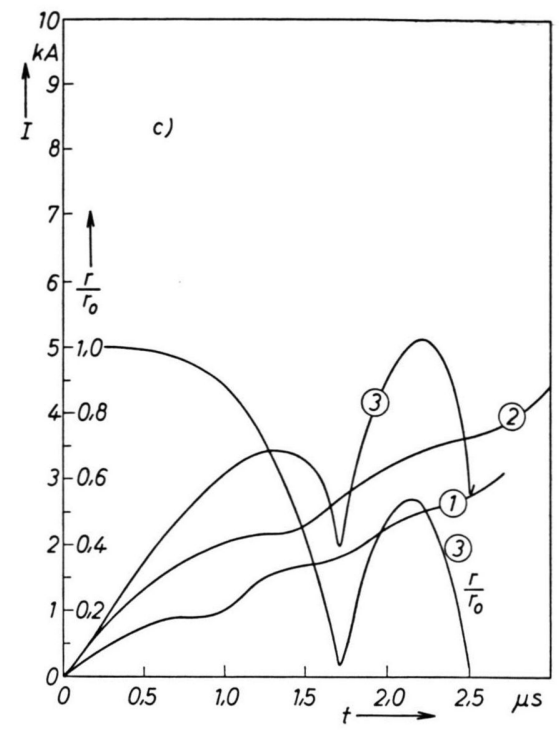

Abb. 15 c. Gemessener und berechneter Stromverlauf sowie berechneter Verlauf des relativen Pinchdurchmessers $r / r_{0}$ für $4 \mathrm{kV}$; 0,02 Torr; 0 Г. 1 Experimentell, ohne Vorentladung; 2 experimentell, mit Vorentladung; 3 theoretisch, $\gamma=1$.

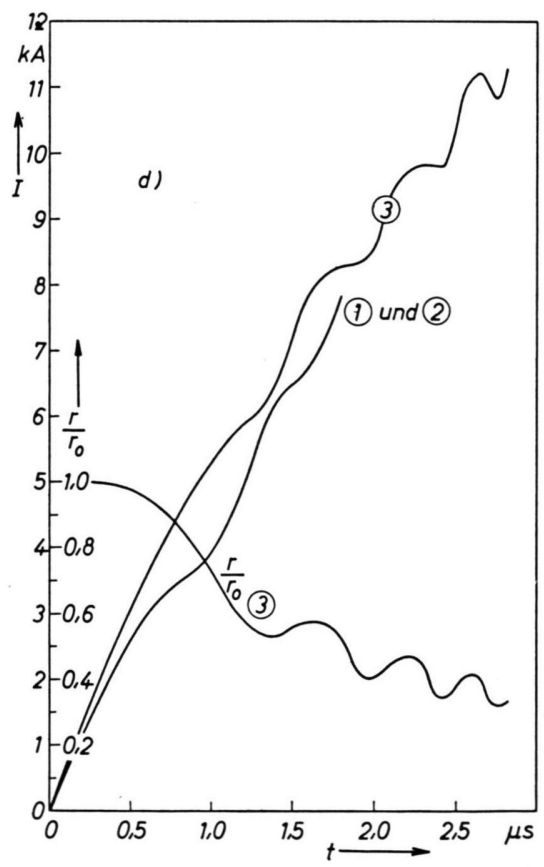

Abb. 15 d. Gemessener und berechneter Stromverlauf sowie berechneter Verlauf des relativen Pinchdurchmessers $r / r_{0}$ für $8 \mathrm{kV}$; 0,02 Torr; $400 \Gamma$. 1 Experimentell, ohne Vorentladung; 2 experimentell, mit Vorentladung; 3 theoretisch, $\gamma=1$.

verwendeten schwachen Vorionisierung zunächst sehr klein ist und im Verlauf der ersten $\mu$ s stark anwächst, wurde versucht, durch eine starke Vorionisierung (Vorentladung, vgl. I, 5) definiertere Verhält- 


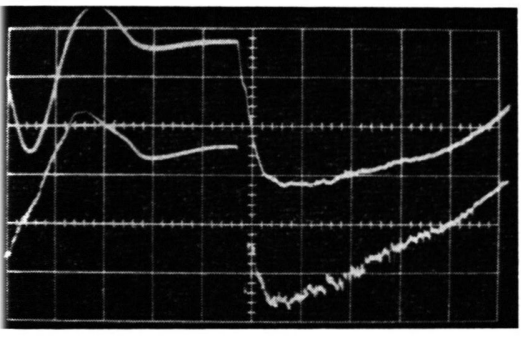

$\boldsymbol{a}$

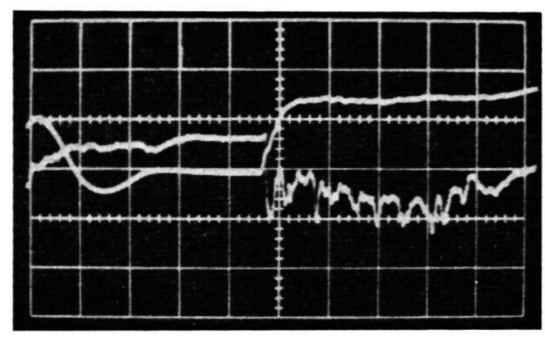

b

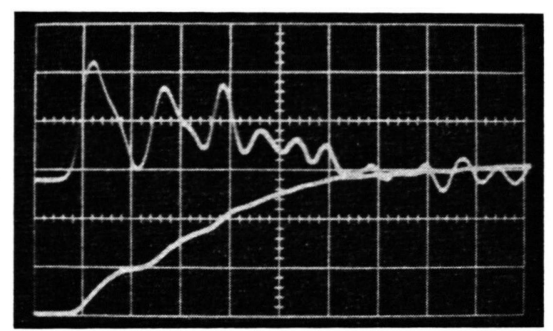

$\boldsymbol{c}$

Abb. 16. Oszillogramm einer Entladung mit Vorentladung $\left(U_{00}=10 \mathrm{kV} ; U_{0}=8 \mathrm{kV} ; 0,05\right.$ Torr; $\left.0 \Gamma\right)$. a) Oberer Strahl (nach unten) : Plasmastrom $J_{2}, 5 \mathrm{kA} / \mathrm{cm} ; 10 \mu \mathrm{s} / \mathrm{cm}$, unterer Strahl (nach unten) : Ringspannung $U, 2 \mathrm{kV} / \mathrm{cm}$. b) Oberer Strahl (nach oben): Primärstrom $J_{1}, 10 \mathrm{kA} / \mathrm{cm}, 10 \mu \mathrm{s} / \mathrm{cm}$, unterer Strahl (nach unten) : $\mathrm{H}_{\beta}$-Intensität. c) Oberer Strahl (nach oben) : $\mathrm{d} J_{1} / \mathrm{d} t, 2,2 \cdot 10^{9} \mathrm{As}^{-1} / \mathrm{cm} ; 1 \mu \mathrm{s} / \mathrm{cm}$, unterer Strahl (nach oben) : $J_{1}, 5 \mathrm{kA} / \mathrm{cm}$. Das Oszillogramm Abb. $16 \mathrm{c}$ zeigt den Beginn der Hauptentladung.

nisse zu schaffen. Der Verlauf des Stromes bei starker Vorionisierung ist in den Diagrammen Abb. 15 eingezeichnet. In den Oszillogrammen der Abb. 16 ist der Verlauf von $I_{1}, \mathrm{~d} I_{1} / \mathrm{d} t, U, I_{2}$ und $\mathrm{H}_{\beta}$-Intensität bei einer Entladung mit Vorentladung aufgezeichnet.

\section{Diskussion}

Die angewendete Theorie weicht in den folgenden Punkten von der experimentellen Wirklichkeit $a b$ :

a) Bei schwacher Vorionisation ist die an der Bewegung beteiligte Masse nicht zeitlich konstant.

b) Die elektrische Leitfähigkeit ist endlich und kann von Ort und Zeit abhängen.

c) Energie kann an die Toruswand geleitet werden, z. B. durch Teilchendiffusion und runaway-Elektronen.

d) Der Strom wird nicht an der Oberfläche eines Zylinders, sondern über den ganzen Querschnitt verteilt fließen. Dies folgt schon aus b); darüber hinaus kann man sich vorstellen, daß auch hinter einem sich zusammenziehenden Oberflächenstrom endlicher Dicke hinreichend leitendes Plasma zurückbleibt, um Ströme dort fließen zu lassen.

e) Die radiale Geschwindigkeit $\mathrm{d} r / \mathrm{d} t$ braucht nicht proportional zu $r$ zu sein. In der Tat wurde das sog. "Schneepflugmodell“ auch schon zur Beschreibung eines sich zusammenziehenden Plasmazylinders verwendet (z. B. ${ }^{31}$ ).

f) Die Geometrie ist toroidal und nicht linear.

Mit Hilfe der ersten vier der angeführten Punkte ist es weitgehend möglich, die Abweichungen der gemessenen von den gerechneten Kurven zu erklären. Im folgenden werden die bei bestimmten Bedingungen auftretenden Erscheinungen beschrieben und mit der Theorie verglichen.

\subsection{Pinch ohne Vorionisation}

Da die zur Erreichung wesentlicher Ionisationsgrade notwendige Zeit etwa 1 bis $3 \mu$ s beträgt, beteiligt sich an der ersten Schwingung nur ein kleiner Bruchteil der Masse; an den folgenden Schwingungen wird dann mehr Masse beteiligt sein infolge des inzwischen erreichten hohen Ionisationsgrades. So erklärt sich, daß der Zeitpunkt der ersten Kontraktion schon bei etwa $0,5 \mu \mathrm{s}$, statt, wie in der Rechnung, bei etwa $1,0 \mu$ s liegt, während die gemessenen Abstände der folgenden Kontraktionen einigermaßen mit den gerechneten übereinstimmen. Der Strom wird über den Querschnitt verteilt sein wegen der schlechten Leitfähigkeit. Der Oнмsche Widerstand $R$, ebenso wie die gegenüber einem Oberflächenstrom höhere Induktivität, haben einen insgesamt kleineren Strom zur Folge, als er sich aus der Rechnung ergibt. Die gemessenen Amplituden der Stromschwingung sind kleiner, weil dem pulsierenden Oberflächenstrom ein Volumenstrom überlagert ist. Die auf das Plasma wirkende Kraft $\mathfrak{j} \times \mathfrak{B}$ ist also kleiner als die in der Rechnung, woraus man schließen muß, daß auch die an der Bewegung beteiligte Masse kleiner ist, als es einer gerechneten Kurve mit gleicher Frequenz entspricht.

\subsection{Pinch mit Vorionisation}

Wenn man annimmt, daß wegen der Vorionisation die Leitfähigkeit des Plasmas zu Beginn der Entladung dieselbe Größenordnung hat wie z. B. im Strommaximum, dann ist die Zeit, innerhalb derer ein Magnetfeld wesentlich durch den Torus diffundiert, etwa $1 \mu \mathrm{s}$. Daraus folgt, da $\beta$ man innerhalb von etwa $0,5 \mu$ s ungefähr mit Oberflächenströmen rechnen kann, daß sich dann aber ein Volumenstrom ausbilden wird. Die Vorionisation wird also die In- 
duktivität während der ersten Mikrosekunde verkleinern. Aus diesem Grund und weil die Leitfähigkeit größer ist, wird der Strom stärker ansteigen als im Fall der ganz schwachen Vorionisation. Man sieht z. B. aus Abb. 15 b und 15 c, daß mit Vorionisation der Zeitpunkt der ersten Kontraktion und der zugehörige mittlere Stromwert mit den gerechneten Werten ungefähr übereinstimmen. Den Unterschied zwischen den gemessenen und gerechneten Amplituden der Schwingung wird man wieder mit Volumenströmen erklären, ebenso den Unterschied in den Frequenzen: Wie im Fall ohne Vorionisation ist die treibende Kraft $\mathfrak{j} \times \mathfrak{B}$ kleiner als in der Rechnung, die Masse aber ungefähr gleich - so kommt eine Frequenz zustande, die kleiner ist als die theoretische.

\subsection{Pinch mit überlagertem $B_{z}$ - Feld}

Erzeugt man im Torus vor der Entladung ein seelenparalleles, quasistationäres Magnetfeld („, $B_{z^{-}}$ Feld“), so werden die Amplituden der Pinch-Oszillationen, sowohl in der Theorie wie im Experiment, sehr verringert, während die Frequenz sich nicht ändert. Weil die gemessenen Amplituden so klein sind, läßt sich ein Vergleich zwischen Theorie und Experiment nur noch schwer durchführen. Die Schwingungen sind nur noch schwach sichtbar - eigentlich nur noch in $\operatorname{der} \mathrm{d} I / \mathrm{d} t$-Kurve - und zeigen in Abhängigkeit von den Entladungsparametern eine uneinheitliche Struktur. So hat z. B. bei kleinen Drucken die Vorionisation keinen Einfluß auf den Stromverlauf (Abb. $15 \mathrm{~d}$ ). Bei hohen Drucken und hoher Vorionisation dagegen oszilliert der Strom mit einer Frequenz, die etwa viermal kleiner ist als die gerechnete. Dabei treten überlagerte Schwingungen mit der Frequenz auf, die man ohne Vorionisation beobachtete.

\subsection{Drehspiegelkamera-A u f $\mathrm{n}$ ahmen}

In Kap. III, 4 sind die zeitlich aufgelösten Photographien des Plasmas schon beschrieben worden. Hier interessieren wir uns speziell für einen quantitativen Zusammenhang zwischen den Leuchterscheinungen während etwa der ersten $3 \mu$ s nach Beginn der Entladung und den Stromoszillationen. Die besprochenen Bilder sind alle ohne starke Vorionisation aufgenommen worden.

Bei $p_{0}=0,05$ Torr hält die über den ganzen Querschnitt verteilte Leuchterscheinung ungefähr $0,5 \mu \mathrm{s}$ lang an (Abb. 17). Die anschließende Kontraktion erfolgt etwa während $0,2 \mu \mathrm{s}$. Das erste Stromminimum, das in unserem Modell mit der ersten maximalen Kompression praktisch zusammenfällt, wird knapp $1 \mu$ s nach Strombeginn beobachtet (Abb. 15 b). Ganz schwach ist noch während der nächsten Mikrosekunde eine pulsierende Struktur zu erkennen, die ebenfalls zeitlich den beiden folgenden Stromschwankungen zugeordnet werden kann. Die Übereinstimmung zwischen Bildstruktur und Stromoszillogramm ist nicht in allen Fällen so gut. Bei $4 \mathrm{kV}, 0,1$ Torr, $0 \Gamma$ z. B. bleibt der Torus nach der Zündung etwa $1,5 \mu$ s lang hell, während das erste Stromminimum schon bei $0,8 \mu$ s erscheint.

\section{Zusammenfassung}

Die Übereinstimmung zwischen dem gerechneten und dem gemessenen Stromverlauf zu Beginn der Entladung und den Drehspiegelkamera-Aufnahmen ist hinreichend, um sicher zu sein, daß die beobachteten Stromoszillationen durch Schwingungen des komprimierten Plasmaschlauches hervorgerufen werden. Die Differenzen zwischen den experimentellen und den theoretischen Daten lassen sich im allgemeinen erklären. Es bleiben in einigen Fällen Diskrepanzen, die nicht leicht zu verstehen sind. Vergleicht man die vorliegende Untersuchung z. B. mit der von Lehner, so scheint dort die Übereinstimmung zwischen Experiment und Theorie besser zu sein als hier. Allgemein wird man eine um so bessere Übereinstimmung erwarten, je besser die folgenden vier Bedingungen erfüllt sind:

a)

$$
\frac{L_{\mathrm{a}}}{2 l \ln r_{0} / r}<10^{-9} \mathrm{H} \mathrm{cm}^{-1}
$$

( $L_{\mathrm{a}}=$ gesamte zeitlich konstante Induktivität in Henry, $l=$ Länge des Entladungsgefäßes in $\mathrm{cm}$, $r_{0}, r=$ Radius des Entladungsgefäßes bzw. des Plasmas bei maximaler Kompression).

Diese Bedingung bedeutet einfach, daß der durch die Plasmakontraktion hervorgerufene Induktivitätsanteil größer ist als die zeitlich konstante Induktivität $\left(L_{\mathrm{a}}=L_{\mathrm{z}}+2 \cdot 10^{-9} l \ln \left(R / r_{0}\right)\right.$, wobei $L_{\mathrm{z}}$ in Henry die Induktivität der Zuleitung und $R$ der Radius des Metallmantels ist). Wenn die Bedingung nicht erfüllt ist, werden die Schwingungen so klein, daß es schwierig ist, sie von Nebeneffekten gut zu unterscheiden. Im T-1 ist

$$
\frac{L_{\mathrm{a}}}{2 l \ln r_{0} / r} \approx 2 \cdot 10^{-9} \mathrm{H} \mathrm{cm}^{-1},
$$


bei LeHNER ist der entsprechende Wert $1,6 \cdot 10^{-10}$.

b) $\quad T \equiv \sigma_{\mathrm{p}} r_{0}^{2} \cdot 10^{-9} \gg \tau=6 \sqrt[4]{M} \sqrt{\frac{r_{0}}{U_{0} / L_{\mathrm{a}}}}$

oder

$$
\frac{U_{0}}{L_{\mathrm{a}}} \gg \frac{2 \cdot 10^{16}}{\sigma_{\mathrm{p}}^{2} r_{0}^{2}} \sqrt{p_{0}} \text { für } \mathrm{H}_{2} \text {. }
$$

$\left(\sigma_{\mathrm{p}}=\right.$ elektrische Leitfähigkeit in $\Omega^{-1} \mathrm{~cm}^{-1}, M=$ Masse des Plasmas pro cm Gefäßlänge in g, $U_{0}=$ Ladespannung des Kondensators in $\mathrm{V}, p_{0}=$ Anfangsdruck des Gases in Torr, $\tau$ ist etwa gleich der Periode der Plasmaoszillationen [vgl. ${ }^{29}$ ]).

Wenn die Bedingung erfüllt ist, kann man mit Oberflächenströmen rechnen; bei Gleichheit von $T$ und $\tau$ ist beim Zeitpunkt der ersten Kontraktion $(t=\tau)$ die Dicke der stromführenden Schicht gleich $r_{0} / 3$. Bei LehNer ist

$\frac{U_{0}}{L_{\mathrm{a}}} \approx 10^{12} \mathrm{~A} \mathrm{~s}^{-1}$ und $\frac{2 \cdot 10^{16}}{\sigma_{\mathrm{p}}{ }^{2} r_{0}{ }^{2}} \sqrt{p_{0}}=6 \cdot 10^{9} \mathrm{~A} \mathrm{~s}^{-1}$

bei $p_{0}=0,1$ Torr, die Ungleichung ist also erfüllt. Im T-1 ist

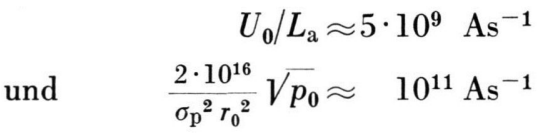

bei 0,1 Torr und Vorionisation. Man sieht, daß im T-1 selbst bei Vorionisation die Bedingung für Oberflächenströme nicht erfüllt ist.

c)

$$
\frac{M r_{0}}{\tau^{2}} \gg p_{0}\left(r_{0} / r\right)^{2 \varkappa}
$$

$\left(\varkappa=c_{p} / c_{v}\right.$ Verhältnis der spezifischen Wärmen des Plasmas).

Setzt man $\varkappa=2$ und $r_{0} / r=3$, so folgt, wieder für Wasserstoff,

$$
U_{0} / L_{\mathrm{a}} \gg 5 \cdot 10^{6} \sqrt{p_{0}} / r_{0} \mathrm{~A} \mathrm{~s}^{-1} .
$$

Diese Bedingung bedeutet, daß die Massenträgheit eine entscheidende Rolle in der Kräftebilanz spielt. Ist die Bedingung nicht erfüllt, so bleibt $\mathrm{d} I / \mathrm{d} t$ immer größer Null. Diese Ungleichung ist im T-1 gut erfüllt.

d) $\sqrt{L_{\mathrm{a}} C} \gg \tau$ oder $\quad U_{0} \gg 2 \cdot 10^{-2} \frac{r_{0}{ }^{2}}{C} \sqrt{p_{0}}$

( $C=$ Kapazität der Kondensatorbatterie in Farad).

Die Ungleichung gilt wieder für Wasserstoff. Diese Bedingung verlangt, daß die Frequenz der Plasmaoszillationen erheblich größer ist als die der Entladung, sie ist im T-1 erfüllt.

Es läßt sich verstehen, daß die Übereinstimmung zwischen Theorie und Experiment im T-1 nicht so gut ist wie bei LeHNer, wenn man weiß, daß die beiden Bedingungen a) und b) dort gut und hier nicht erfüllt sind. Mit Hilfe der vier Bedingungen ist auch zu erklären, warum z. B. in der größeren und langsameren Torus-Pinchentladung des MPI („Mimikry“) Stromoszillationen überhaupt nicht mehr zu beobachten sind.

\section{Energiebilanz des quasistationären Zustandes}

\section{Leistungszufuhr}

Die dem Plasma zugeführte elektrische Leistung $W=I^{2} R_{\mathrm{p}}$ ist für den größten Teil der Entladung gut bekannt, da, wie gezeigt wurde, der OHмsche Plasmawiderstand $R_{\mathrm{p}}$ während langer Zeiten eine bekannte und sogar zeitlich konstante Größe ist. Abb. 18 zeigt als Beispiel den Verlauf von $W(t)$ für einen bestimmten Fall.

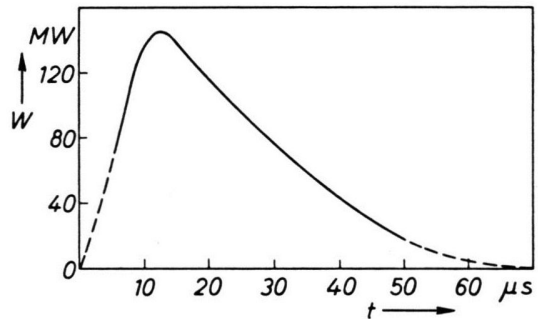

Abb. 18. Dem Plasma zugeführte elektrische Leistung bei $8 \mathrm{kV} ; 0,2$ Torr; $800 \Gamma$.

Die Kurve wurde für kleine Zeiten, wo wir $R_{\mathrm{p}}$ nicht mehr gut kennen, nach Null extrapoliert. Die entsprechenden Kurven wurden für eine Reihe anderer Fälle ermittelt. Es zeigte sich, daß das Plasma während rund $40 \mu$ s mit einer Leistung geheizt wird, die, je nach den Parameterwerten, zwischen 10 und $100 \mathrm{eV}$ pro Mikrosekunde und Elektron liegt. Die aus der Leitfähigkeit bestimmten Elektronentemperaturen, die, wie wir gesehen haben, sich während dieser Zeit praktisch nicht ändern, liegen zwischen 3 und $6 \mathrm{eV}$. Das Plasma gibt die Energie ebenso schnell wieder ab wie es sie aufnimmt. Dabei haben die Verlustprozesse offenbar die Eigenschaft, von der Temperatur oder der Leistung so stark abzuhängen, daß $T_{\mathrm{e}}$, unabhängig von der Heizleistung, im wesentlichen unverändert bleibt.

Auf der anderen Seite wissen wir, daß das (schwach ionisierte) Plasma die ihm zugeführte Energie im wesentlichen nicht wieder abgibt, sondern zur Ionisation benutzt. Einen Überblick über diese Zusammenhänge gibt die Tab. 4. 


\begin{tabular}{|c|c|c|c|c|c|c|c|}
\hline 1 & 2 & 3 & 4 & 5 & 6 & 7 & 8 \\
\hline $\begin{array}{c}P \\
\text { Torr }\end{array}$ & $\begin{array}{l}U_{\mathrm{o}} \\
\mathrm{kV}\end{array}$ & $\begin{array}{c}B_{\mathrm{Z}} \\
\Gamma\end{array}$ & $\begin{array}{c}E_{\text {ion }} \\
\text { Ws }\end{array}$ & $\begin{array}{c}E\left(t_{\mathrm{M}}\right) \\
\text { Ws }\end{array}$ & $\begin{array}{c}W_{\max } \\
\mathrm{MW}\end{array}$ & $\begin{array}{c}W_{\max } / 2 N_{\mathrm{e}} \\
\text { eV pro } \mu \mathrm{s} \\
\text { u. Elektron }\end{array}$ & $\begin{array}{c}T_{\mathrm{e}} \\
\mathrm{eV}\end{array}$ \\
\hline 0,02 & 4 & 800 & 17 & 10 & 36 & 23 & 5,2 \\
& 8 & 800 & & 15 & 104 & 65 & 3,5 \\
0,05 & 4 & 800 & 42 & 15 & 36 & 9 & 4,6 \\
& 6 & 800 & & 30 & 75 & 19 & 4 \\
& 8 & 0 & & 80 & 100 & 25 & 4 \\
0,1 & 8 & 800 & & 45 & 120 & 30 & 4 \\
& 4 & 800 & 85 & 100 & 36 & 4,5 & 5,2 \\
0,2 & 8 & 800 & & 120 & 126 & 16 & 4 \\
& 4 & 0 & 170 & 10 & 31 & 2 & 5,2 \\
& 8 & 800 & & 210 & 146 & 10 & 5 \\
& 8 & 0 & 340 & 450 & 144 & 4,3 & 5,2 \\
\hline
\end{tabular}

Tab. 4. Größen der Energiebilanz. Spalte 4 enthält die Energie $E_{\text {ion }}$, die notwendig ist, um das ganze Gas im Torus zu ionisieren und auf $5 \mathrm{eV}$ zu heizen. Spalte 5 gibt die (roh berechneteten) Werte des Integrals

$$
E\left(t_{\mathrm{M}}\right)=\int_{0}^{t_{\mathrm{M}}} W(t) \mathrm{d} t
$$

wobei für $W$ die graphisch extrapolierten Werte benutzt wurden und $t_{\mathrm{M}}$ wieder die in Tab. 1 aufgeführten Zeiten sind, bei denen das erste Maximum in der $\mathrm{H}_{\beta}$-Intensität auftritt. In Spalte 6 werden der gemessene Maximalwert und in Spalte 7 ein rỏher Mittelwert von $W$ (während ungefähr $40 \mu \mathrm{s}$ ), dividiert durch die Gesamtzahl der Elektronen $N_{\mathrm{e}}$, aufgeführt. Spalte 8 enthält die aus der Leitfähigkeit nach SPITzER ermittelten Temperaturen.

Man sieht, daß die bis zur vollständigen Ionisation vom Plasma aufgenommene Energie dieselbe Größenordnung hat wie sein Energieinhalt. Es liegt an der Ungenauigkeit der Abschätzung von $E\left(t_{\mathrm{M}}\right)$, daß sich diese Größe in einigen Fällen sogar kleiner ergab als $E_{\text {ion }}$ [in Wirklichkeit ist natürlich immer $\left.E_{\text {ion }} \leq E\left(t_{\mathrm{M}}\right)\right]$.

Zusammenfassend läßt sich sagen, daß das Plasma bis zur vollständigen Ionisation etwa $45 \mathrm{eV}$ pro $\mathrm{H}_{2}$ Molekül aufnimmt, ohne dabei wesentlich Energie $\mathrm{zu}$ verlieren. $45 \mathrm{eV}$ ist ungefähr die Energie, die aufgewendet werden muß, um das $\mathrm{H}_{2}$-Molekül in 2 Protonen und 2 Elektronen mit $5 \mathrm{eV}$ Temperatur zu überführen. Danach aber, während praktisch der gesamten Entladung, werden dem Plasma bis $100 \mathrm{eV}$ pro Mikrosekunde und Elektron, insgesamt also bis einige keV pro Elektron, zugeführt, während die Temperatur sich um weniger als $1 \mathrm{eV}$ ändert.

\section{Elektronentemperatur und Energieverlust}

Bei 0,2 und 0,4 Torr Anfangsdruck (u. $U_{0}=8 \mathrm{kV}$ ) wurde festgestellt (Kap. IV, 4, 3), daß der von Spitzer angegebene „klassische“ Zusammenhang zwischen Elektronentemperatur und elektrischer Leitfähigkeit in unserem Plasma weitgehend Gültigkeit hat. Im folgenden soll angenommen werden, daß dieser Zusammenhang ebenso bei kleineren Drucken (und Spannungen) besteht. So können wir jeder Entladung eine Elektronentemperatur $T_{\mathrm{e}}$ zuordnen, deren Werte zwischen $3,3 \mathrm{eV}$ (bei 0,02 Torr, $8 \mathrm{kV}$, $0 \Gamma)$ und $6 \mathrm{eV}(0,4$ Torr, $8 \mathrm{kV}, 800 \Gamma)$ liegen. Die Abhängigkeit von $T_{\mathrm{e}}$ von den Entladungsparametern kann den Diagrammen der Abb. 9 entnommen werden, wenn man berücksichtigt, daß $T_{\mathrm{e}} \sim \sigma^{2 / 3}$ ist. Im folgenden soll diese Abhängigkeit kurz diskutiert werden.

a) $T_{\mathrm{e}}$ nimmt mit steigendem Druck $p_{0}$ zu, und zwar um etwa $60 \%$ von 0,02 bis 0,04 Torr, obwohl die spezifische Leistungszufuhr hierbei von $65 \mathrm{eV}$ auf 4,6 eV pro Mikrosekunde und Elektron abnimmt. Die Elektronentemperatur wird vollständig von den energieverzehrenden Prozessen bestimmt und ist unabhängig von der Energiezufuhr. Die Verluste nehmen mit sinkendem Druck oder einer korrelierten Größe (z. B. steigender spezifischer Leistungszufuhr) stark zu.

b) Mit steigender Kondensatorladespannung $U_{\mathbf{0}}$ nimmt $T_{\mathrm{e}} \mathrm{ab}$, und zwar um $20 \%$ zwischen $4 \mathrm{kV}$ und $8 \mathrm{kV}$. Hieraus folgt, daß die Verluste mit der elektrischen Feldstärke oder mit dem Strom oder mit der spezifischen Leistungszufuhr ansteigen und den Temperaturwert bestimmen. 
c) Die Zunahme der Leitfähigkeit mit $B_{z}$ hat vermutlich nichts mit einer Temperaturzunahme zu tun, sondern läßt sich innerhalb der Fehlergrenzen durch die Abhängigkeit von $\sigma$ von der Richtung des resultierenden Magnetfeldes erklären (vgl. z. B. SPItzer).

\section{Verlustmechanismen}

3.1. Magnetohydrodynamische Beschreibung der Entladung

( Pinchmodell)

Die Plasmagleichungen des sogenannten Zweiflüssigkeitsmodells haben, wenn man sie auf die vorliegende Entladung (für $B_{z}=0$ ) anwendet, eine stationäre Lösung (vgl. SpITzer ${ }^{17}$ ). Diese Lösung beschreibt eine zylindrische Plasmasäule, durch die ein Strom fließt, dessen Wechselwirkungskraft mit seinem eigenen Magnetfeld $\mathfrak{j} \times \mathfrak{B}$, dem Gradienten des Teilchendruckes, $\operatorname{grad} n k T$, überall das Gleichgewicht hält. Einen nicht verschwindenden Teilchenfluß gibt es nur längs der Achse, die Teilchendiffusion radial nach außen wird aufgehoben durch eine Drift nach innen.

Nimmt man der Einfachheit halber eine vom Ort unabhängige Temperatur $T$ (Mittelwert zwischen Protonen- und Elektronentemperatur) und damit eine konstante elektrische Leitfähigkeit $\sigma$ an, so ergibt sich ein Volumenstrom konstanter Dichte $j$ und ein parabelförmiges Druckprofil $p$ :

$$
\begin{array}{ll}
p=p_{0}-\pi j^{2} r^{2} & \text { für } \quad r<r_{1}, \\
p=0 \quad \text { für } \quad r \geq r_{1}, \\
p_{0}=n_{0} k T=\pi j^{2} r_{1}{ }^{2} .
\end{array}
$$

Hieraus folgt

$$
r_{1}^{2}=\frac{\sqrt{2 N k T}}{\pi \sigma F}
$$

$N=$ Teilchenzahl $/ \mathrm{cm} ; F=$ elektrische Feldstärke.

\begin{tabular}{|c|c|c|c|c|c|}
\hline $\begin{array}{c}p_{0} \\
\text { Torr }\end{array}$ & $\begin{array}{c}U_{0} \\
\mathrm{kV}\end{array}$ & $\begin{array}{c}T \\
{ }^{\circ} \mathrm{K}\end{array}$ & $\begin{array}{c}\sigma \\
\Omega^{-1} \mathrm{~cm}^{-1}\end{array}$ & $\begin{array}{c}F \\
\mathrm{~V} \mathrm{~cm}^{-1}\end{array}$ & $\begin{array}{c}2 r_{1} \\
\mathrm{~cm}\end{array}$ \\
\hline 0,4 & 4 & $6 \cdot 10^{4}$ & 130 & 10 & 5,2 \\
0,2 & 8 & $5 \cdot 10^{4}$ & 100 & 20 & 3,8 \\
0,02 & 8 & $4 \cdot 10^{4}$ & 60 & 20 & 2,4 \\
\hline
\end{tabular}

Tab. 5. Plasmadurchmesser bei verschiedenen Entladeparametern.

In Tab. 5 sind für drei Fälle die sich aus Gl. (15) ergebenden Durchmesser $2 r_{1}$ der Plasmasäule angegeben.
Die aus Gl. (15) folgenden Plasmadurchmesser sind ungefähr so groß wie der Rohrdurchmesser $(4 \mathrm{~cm})$. Man könnte dies für eine Erklärung der Energieverluste im Rahmen dieses Modells halten: Das Plasma ist wegen seiner zu großen räumlichen Ausdehnung in Kontakt mit der Wand.

Gegen diese Erklärung spricht aber, daß die Verluste mit steigendem Druck und sinkender Spannung ab- statt zunehmen, wie man aus Gl. (15) erwarten würde. Außerdem folgt aus Gl. (15), daß $r_{1} \sim T^{-1 / 2}$ ist. Zugeführte Energie sollte den Pinchradius verkleinern und damit die Verluste verringern, was ein weiteres Zusammenziehen des Plasmas zur Folge haben sollte. Das Plasma sollte unter diesen Umständen die Tendenz haben, sich von der Wand zu lösen.

Eine andere Erklärung für die Energieverluste im Rahmen der magnetohydrodynamischen Theorie wären die magnetohydrodynamisch verständlichen, sog. makroskopischen Instabilitäten. Aus den bestehenden Stabilitätstheorien (vgl. z. B. ${ }^{34,35}$ ) und einigen Experimenten ${ }^{4,11,36,37}$ wird man vermuten, daß das Plasma im Torus nicht im stabilen Gleichgewicht ist - dies um so mehr, als das "Stabilisierungsfeld“ $\left(B_{z}\right.$-Feld) bei unseren Leitfähigkeiten innerhalb von etwa $2 \mu$ s durch das ganze Plasma diffundieren kann. Auf den Drehspiegelkameraaufnahmen sind Anzeichen von Instabilitäten zu erkennen. Eine genauere Prüfung dieser Vermutung wäre aber erst durch eine Messung der Stromdichte in Abhängigkeit von Ort und Zeit mit Hilfe von Magnetfeldsonden möglich. Solche Messungen können sinnvoll am T-1 nicht durchgeführt werden, weil einmal die Sondenabmessungen relativ zum Plasma nicht klein genug sind. Außerdem könnte nur am Pumpstutzen gemessen werden, wo die Symmetrie der Entladung ziemlich gestört ist.

Einen Hinweis darauf, daß einfache magnetohydrodynamische Instabilitäten nicht, oder zumindest nicht allein für den Energieverlust verantwortlich sind, geben die Messungen an anderen toroidalen Entladungen. Im ZETA $^{7}$ und im PERHAPSA$\mathrm{TRON}^{3}$ z. B. wurde mit Sondenmessungen festge-

34 R. J. TAYler, Proc. Phys. Soc., Lond. B 70, 31 [1957].

35 B. R. Suydam, Proc. Gen. Conf. 31, 157 [1958]

36 L C. Burkhardt u. R. H. Lovberg, Proc. Gen. Conf. 32, 29 [1958]

37 D. H. Birdsall, S. A. Colgate u. H. P. Furth, Proc. Uppsala Conf. 888 [1959]. 
stellt, daß sich der Strom im wesentlichen stabil ${ }^{38}$ in der Mitte des Entladungsgefäßes hält. Aber auch dort, wie bei allen anderen $z$-Pinchen, erreicht die Elektronentemperatur nur die Größenordnung $10 \mathrm{eV}$ bei vergleichbarer spezifischer Leistungszufuhr (vgl. Kap. VII, Tab. 6).

\subsection{Pumpstutzen}

Der Einfluß des Pumpstutzens auf den Entladungsverlauf wurde untersucht. Hierzu wurde das Loch, wo der Stutzen in der Toruswand ansetzt, mit einer (verglasten) Metallscheibe praktisch ganz verschlossen. Strom- und Spannungsverlauf blieben hierdurch bis auf etwa $5 \%$ unverändert. Da dies das Maß der Reproduzierbarkeit ist, konnte ein Einfluß eines Loches in der Wand nicht festgestellt werden.

\subsection{Verunreinigungs strahlung}

Die von Verunreinigungen abgestrahlte Leistung schätzen wir ab aus der Abb. 19, die einer Arbeit von KNoRR ${ }^{39}$ entnommen wurde.

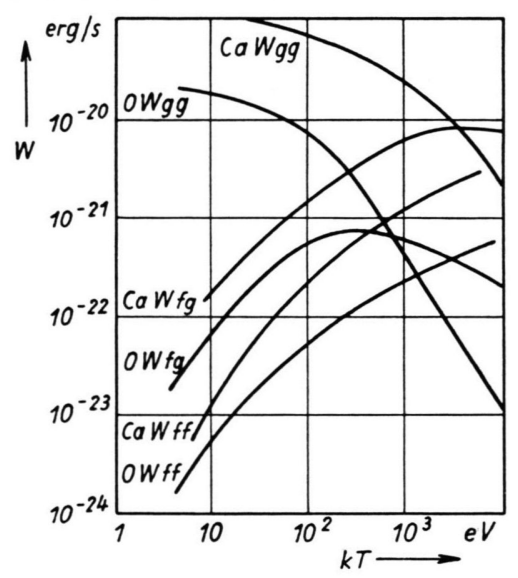

Abb. 19. Ausstrahlung $\mathrm{W}$ von Sauerstoff und Calcium pro Atom und freies Elektron. $\mathrm{ff}=$ frei-frei, $\mathrm{fg}=$ frei-gebunden, $\mathrm{gg}=$ gebunden-gebunden.

Entsprechend dem aus den Spektrogrammen geschätzten Wert wird als Dichte der Verunreinigung $10^{13}$ Sauerstoffatome pro $\mathrm{cm}^{3}$ angenommen. Dann ergibt sich für $T=10 \mathrm{eV}$ die gesamte abgestrahlte

38 Mit Sonden ist ein im großen und ganzen stabiles und ruhiges Plasma in der Mitte des Entladungsgefäßes festzustellen. Dabei sind aber den Sondensignalen nicht reproduzierbare, schnelle Fluktuationen von der Größenordnung $10 \%$ überlagert. Von experimenteller Seite wird dieser $\mathrm{Zu}$ -
Leistung $\mathrm{zu}$

$$
\begin{aligned}
& W_{\mathrm{v}}=8 \cdot 10^{4} \mathrm{~W} \text { bei } p_{0}=0,02 \text { Torr }, \\
& W_{\mathrm{v}}=8 \cdot 10^{5} \mathrm{~W} \text { bei } p_{0}=0,2 \text { Torr, } \\
& W_{\mathrm{v}}=1,6 \cdot 10^{6} \mathrm{~W} \text { bei } p_{0}=0,4 \text { Torr. }
\end{aligned}
$$

Selbst im ungünstigsten Fall ist $W_{\mathrm{v}}$ noch ein bis zwei Größenordnungen kleiner als die dem Plasma zugeführte Leistung. Außerdem nehmen die Strahlungsverluste mit zunehmender Temperatur (bis einige $100 \mathrm{eV}$ ) ab und würden daher einem Temperaturanstieg nicht im Wege stehen. Schließlich nimmt die Strahlung mit steigendem Druck zu, während die beobachteten Verluste mit steigendem Druck abnehmen. Die Strahlung von Verunreinigungen kann also als für den Energiehaushalt wesentlicher Prozeß ausgeschlossen werden.

\subsection{Röntgen-Strahlen und runaway-E $l$ e $k$ - tronen}

Die während der Entladung emittierten RöNTGENStrahlen (für $h v>3 \mathrm{keV}$ ) wurden als Funktion der Zeit und der Energie mit Szintillationszählern gemessen ${ }^{40}$. Diese Röntgen-Quanten werden erzeugt beim Auftreffen sog. runaway-Elektronen auf die Wand, d. h. solcher Elektronen, die aus der thermischen Geschwindigkeitsverteilung ausgekoppelt wurden und im elektrischen Feld „freifallend“ relativ hohe Energien erreicht haben.

Ungefähr $2 \mu$ s nach Strombeginn wird ein kurzer Röntgen-Puls beobachtet (Dauer etwa $1 \mu \mathrm{s}$ ), der einer Kathodenstrahlleistung von etwa $1 \mathrm{MW}$ entspricht (falls die Annahme richtig ist, daß überall auf die Torusoberfläche gleichviel schnelle Elektronen auftreffen). Während der Dauer der Entladung dagegen ist die von schnellen Elektronen oberhalb $3 \mathrm{keV}$ an die Wand transportierte Leistung etwa 6 Größenordnungen kleiner als die umgesetzte Leistung. Bei Drucken $p_{0}>0,1$ Torr konnte überhaupt keine RöNTGEN-Strahlung mehr nachgewiesen werden. Schnelle Elektronen mit Energien oberhalb $3 \mathrm{keV}$ spielen also sicher für die Energiebilanz keine Rolle. Für den Energiebereich unterhalb $3 \mathrm{keV}$ kann aber keine Aussage gemacht werden. Es ist in diesem Zusammenhang interessant, daß der quantitative Vergleich zwischen dem gemessenen und dem be-

stand gelegentlich „grob stabil“ (stable in the gross sense) genannt.

39 G. KNorr, Z. Naturforschg. 13 a, 941 [1958].

40 U. Grossmann-Doerth u. J. Junker, Proc. Salzburg Conf., Paper 38 [1961]. 


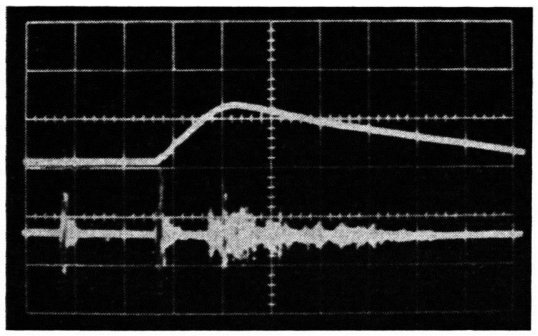

$\boldsymbol{a}$

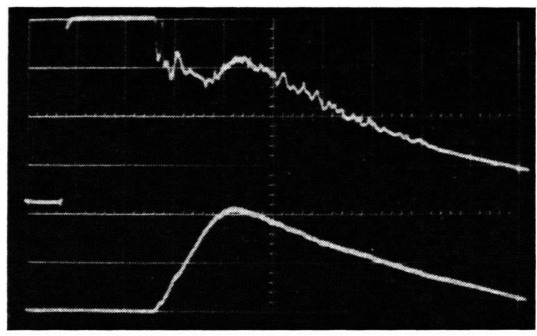

$\boldsymbol{b}$

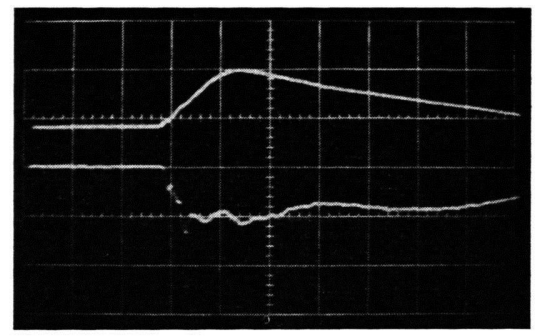

c

Abb. 20. Messung der vom Plasma emittierten Hochfrequenzstrahlung. Entladung bei $4 \mathrm{kV} ; 0,1 \mathrm{Torr} ; 0 \Gamma$. a) Oberer Strahl: $J_{1}, 10 \mathrm{kA} / \mathrm{cm}$; unterer Strahl: $\mathrm{HF}, 0,1 \mathrm{~V} / \mathrm{cm} ; 10 \mu \mathrm{s} / \mathrm{cm}$. b) Oberer Strahl: $U, 1 \mathrm{kV} / \mathrm{cm}$; unterer Strahl: $J_{2}, 5 \mathrm{kA} / \mathrm{cm} ; 10 \mu \mathrm{s} / \mathrm{cm}$. c) Oberer Strahl: $J_{1}, 10 \mathrm{~A} / \mathrm{cm}$; unterer Strahl: (nach unten: $\mathrm{H}_{\beta}$-Intensität; $10 \mu \mathrm{s} / \mathrm{cm}$.

rechneten Verlauf der RöNTGEN-Strahlen-Intensität zu dem Schluß führt, daß ungefähr zur Zeit des ersten $\mathrm{H}_{\beta}$-Maximums Prozesse einsetzen, die die Rate der runaway-Elektronen um etwa $10^{6}$ gegenüber dem theoretisch zu erwartenden Wert vermindern. Möglicherweise sind diese Prozesse auch die Ursache für den großen Energieverlust des Plasmas.

\subsection{H o c h f requenz}

Im Frequenzbereich zwischen $1 \mathrm{MHz}$ und $30 \mathrm{MHz}$ wird während der Entladung eine starke Hochfrequenzstrahlung beobachtet. Der zeitliche Verlauf dieser Strahlung wurde in Abhängigkeit von den Entladungsparametern mit einer außerhalb des Torus aufgestellten Dipolantenne (Länge etwa $8 \mathrm{~cm}$ ) gemessen. In der Abb. 20 ist als Beispiel eines der aufgenommenen Oszillogramme wiedergegeben. Die Strahlung ist ziemlich gut reproduzierbar.

Der erste Strahlungspuls wird vom Ignitron bei dessen Zündung emittiert, der zweite erscheint bei Strombeginn (Dauer etwa $4 \mu \mathrm{s}$ ). Dann folgen einige Mikrosekunden relativer Ruhe. Anschließend setzt die Strahlung wieder ein für eine verhältnismäßig lange Dauer. Es scheint ein Zusammenhang mit der Größe des Stromes zu bestehen, der uns ermutigte, eine Korrelation mit „Zweistrominstabilitäten“ (vgl. z. B. ${ }^{41}$ ) zu suchen. Hierzu sind weitere experimentelle Untersuchungen notwendig, die noch nicht abgeschlossen sind.

Die Abhängigkeit der Hochfrequenz von den Entladungsbedingungen entspricht der der Energieverluste: Sie steigt mit zunehmender Spannung und sinkendem Druck. Vom $B_{z}$-Feld scheint sie nur über den Strom abzuhängen. Wenn eine Mikroinstabilität ${ }^{42}$ bei so niedrigen Frequenzen auftritt, ist auch

41 I. B. Bernstern, Phys. Fluids 3, 937 [1960].

42 Man nennt eine Instabilität mikroskopisch, wenn sie vollständig nur erklärt werden kann aus einer Theorie, die die verständlich, daß sie keinen Einfluß auf die elektrische Leitfähigkeit hat. Es erscheint nach dem Gesagten nicht ausgeschlossen, daß infolge von Mikroinstabilitäten die Teilchen ständig zur Wand gelangen und daß dies die Ursache für die exzessiven Energieverluste ist.

\section{Schlußfolgerung}

Die zu Beginn der Entladung im T-1 auftretenden Phänomene lassen sich mit einfachen Theorien hinreichend gut erklären. Nach Erreichen vollständiger Ionisation jedoch verliert das Plasma in so starkem Maße Energie, daß die Elektronentemperatur praktisch konstant auf einem sehr niedrigen Wert bleibt. A.bschätzungen ergeben, daß als Ursache für den Energieverlust nur Plasmainstabilitäten in Frage kommen. Sondenmessungen, die eine Aufklärung über das Auftreten von einfachen magnetohydrodynamischen Instabilitäten geben würden, sind aus technischen Gründen am T-1 nicht sinnvoll. Das Auftreten solcher Instabilitäten ist durchaus möglich. Auf der anderen Seite wurde in größeren Apparaturen derselben Art unter ähnlichen Bedingungen im wesentlichen Stabilität beobachtet, ohne daß die aus Leitfähigkeitsmessungen sich ergebende Elektronentemperatur größenordnungsmäßig höhere Werte als im T-1 erreicht. Tab. 6 gibt einige Ergebnisse der von anderen Laboratorien durchgeführten Untersuchungen des $z$-Pinches wieder. Einige der Zahlen sind nur ungefähre Werte.

In allen zitierten Experimenten wurde mit Magnetfeldsonden "grobe“ Stabilität (vgl. ${ }^{38}$ ) festgestellt. Während Zeiten, die zwischen 10 und $200 \mu \mathrm{s}$ liegen, wird dem Plasma Energie zugeführt mit

Geschwindigkeitsverteilung der Plasmateilchen enthält. Ein Beispiel hierfür ist die sog. Zweistrominstabilität. 


\begin{tabular}{|c|c|c|c|c|c|}
\hline $\begin{array}{l}\text { Apparatur und Standort } \\
\text { (Anmerkung) }\end{array}$ & Geometrie & $\begin{array}{c}\sigma \\
\Omega^{-1} \mathrm{~cm}^{-1}\end{array}$ & $\begin{array}{l}T_{\mathrm{e}} \\
\mathrm{eV}\end{array}$ & $\begin{array}{l}\quad W / N_{\mathrm{e}} \\
\mathrm{eV} / \mu \mathrm{s} \\
\text { u. Elektron }\end{array}$ & Bemerkungen \\
\hline $\begin{array}{l}\text { PERHAPSATRON } \\
\text { S-4, Los Alamos, USA } \\
\text { (vgl. } 3,11,43)\end{array}$ & $\begin{array}{l}\text { Torus } \\
d=14 \mathrm{~cm} \\
D=70 \mathrm{~cm}\end{array}$ & 200 & 6 & 100 & $\begin{array}{l}\text { Fast konstante Leistungszufuhr } \\
\text { über } 50 \mu \text { s. Aus spektroskopi- } \\
\text { schen Messungen } T_{\mathrm{e}} \approx 40 \mathrm{eV} \text {. } \\
\text { Energieverluste erklärt durch } \\
\text { Strahlung mit Wellenlängen } \\
\text { zwischen } 10^{2} \text { und } 10^{3} \AA\end{array}$ \\
\hline $\begin{array}{l}\text { GAMMA PINCH } \\
\text { Livermore, USA } \\
\text { (vgl. 4) }\end{array}$ & $\begin{array}{c}\text { Torus } \\
d=10 \mathrm{~cm} \\
D=40 \mathrm{~cm}\end{array}$ & & 10 & $\begin{array}{l}\text { über } 10^{3} \\
\text { maximal }\end{array}$ & $\begin{array}{l}\text { Keine spektroskopische Bestim- } \\
\text { mung von } T_{\mathrm{e}} \text {. Innerhalb der } \\
\text { ersten Achtelperiode ist Energie } \\
\text { der runaway-Elektronen inner- } \\
\text { halb der Größenordnung der zu- } \\
\text { geführten Energie }\end{array}$ \\
\hline $\begin{array}{l}\text { ZETA } \\
\text { Harwell, England } \\
\text { (vgl. 7) }\end{array}$ & $\begin{array}{l}\text { Metalltorus } \\
d=100 \mathrm{~cm} \\
D=300 \mathrm{~cm}\end{array}$ & & $5 \ldots 30$ & 2 & $\begin{array}{l}\text { Aus spektroskopischen Messun-- } \\
\text { gen } T_{\mathrm{e}}=20 \mathrm{eV} \text {. Aus Mikro- } \\
\text { wellenrauschen folgt auch } \\
T_{\mathrm{e}}=5 \ldots 30 \mathrm{eV}\end{array}$ \\
\hline $\begin{array}{l}\text { SCEPTRE } \\
\text { Aldermaston, England } \\
\text { (vgl. 8, 9, 12) }\end{array}$ & $\begin{array}{l}\text { Metalltorus } \\
d=30 \mathrm{~cm} \\
D=100 \mathrm{~cm}\end{array}$ & & $5 \ldots 10$ & 50 & $\begin{array}{l}\text { Aus spektroskopischen Messun- } \\
\text { gen } T_{\mathrm{e}}=20 \mathrm{eV} \text { unabhängig von } \\
\text { experimentellen Parametern. In- } \\
\text { stabilitätstheorie von WARE soll } \\
\text { Verluste erklären }\end{array}$ \\
\hline $\begin{array}{l}\text { T-1, München } \\
\text { (diese Arbeit) }\end{array}$ & $\begin{array}{c}\text { Torus } \\
d=4 \mathrm{~cm} \\
D=100 \mathrm{~cm}\end{array}$ & $50 \ldots 200$ & $3 \ldots 6$ & $100 \ldots 5$ & $\begin{array}{l}\text { Aus spektroskopischen Messun- } \\
\text { gen } T_{\mathrm{e}}=5 \mathrm{eV} \text {. Keine Sonden- } \\
\text { messungen }\end{array}$ \\
\hline $\begin{array}{l}\text { COLUMBUS S-4 } \\
\text { Los Alamos, USA } \\
\text { (vgl. 36) }\end{array}$ & $\begin{array}{l}\text { Linear } \\
d=13 \mathrm{~cm} \\
L=61 \mathrm{~cm}\end{array}$ & $150 \ldots 800$ & $5 \ldots 12$ & 250 & $\begin{array}{l}\sigma \text { aus Sondenmessungen lokal } \\
\text { bestimmt }\end{array}$ \\
\hline
\end{tabular}

Tab. 6. Untersuchung des $z$-Pinches. Die Elektronentemperatur $T_{\mathrm{e}}$ wurde aus der gemessenen Leitfähigkeit nach der SpITzERFormel ermittelt. $W$ ist die zugeführte Leistung, $N_{\mathrm{e}}$ die Gesamtzahl der Elektronen.

einer Rate, die von 1 bis $10^{3} \mathrm{eV}$ pro $\mu$ s und Elektron reicht; die dabei beobachteten Elektronentemperaturen sind von der Größenordnung $10 \mathrm{eV}$. Die aus der elektrischen Leitfähigkeit, spektroskopischen Messungen und Mikrowellenrauschen ermittelten Elektronentemperaturen stimmen meist ungefähr überein. Die größte Diskrepanz wurde im PERHAPSATRON S-4 gefunden, wo spektroskopische Messungen Temperaturen von etwa $40 \mathrm{eV}$ ergaben, während aus der Leitfähigkeit $T_{\mathrm{e}} \approx 6 \mathrm{eV}$ folgt ${ }^{3,43}$.

43 H. J. Karr, E. A. Knapp u. J. E. Osher, Phys. Fluids 4, 424 [1961].
Als Erklärung für die großen Energieverluste des Plasmas beim $z$-Pinch werden vor allem Plasmainstabilitäten, Verunreinigungsstrahlung und runaway-Elektronen diskutiert (vgl. VI, 3). Zugunsten jeder der drei Möglichkeiten lassen sich gewisse experimentelle Ergebnisse heranziehen. Eine allgemeine Lösung des Problems steht noch aus.

Herrn Professor W. Heisenberg und Herrn Dr. G. v. Gierke danke ich für die Aufnahme in das Max-PlanckInstitut für Physik und Astrophysik und für die Anregung zu dieser Arbeit. Mein Dank gilt ferner Herrn Dr. K. HaIN und Frau G. HaIN sowie der Rechenmaschinengruppe für die Durchführung der numerischen Rechnungen auf der G-2. 